\title{
Methods to Address Confounding and Other Biases in Meta-Analyses: Review and Recommendations
}

\author{
Maya B. Mathur ${ }^{1}$ and Tyler J. VanderWeele ${ }^{2}$ \\ ${ }^{1}$ Quantitative Sciences Unit and Department of Pediatrics, Stanford University, \\ Stanford, CA, USA; email: mmathur@stanford.edu \\ ${ }^{2}$ Department of Epidemiology, Harvard University, Boston, MA, USA
}

To appear in Annual Reviews of Public Health. 2022. 43:1-19

https://doi.org/10.1146/annurevpublhealth-051920-114020

Copyright (C) 2022 by Annual Reviews. All rights reserved

\section{Keywords}

meta-analysis, bias, confounding, observational studies, sensitivity analysis

\section{Abstract}

Meta-analyses contribute critically to cumulative science, but they can produce misleading conclusions if their constituent primary studies are biased, for example by unmeasured confounding in nonrandomized studies. We provide practical guidance on how meta-analysts can address confounding and other biases that affect studies' internal validity, focusing primarily on sensitivity analyses that help quantify how biased the meta-analysis estimates might be. We review a number of sensitivity analysis methods to do so, especially recent developments that are straightforward to implement and interpret and that use somewhat less stringent statistical assumptions than earlier methods. We give recommendations for how these methods could be applied in practice and illustrate using a previously published meta-analysis. Sensitivity analyses can provide informative quantitative summaries of evidence strength, and we suggest reporting them routinely in meta-analyses of potentially biased studies. This recommendation in no way diminishes the importance of defining study eligibility criteria that reduce bias and of characterizing studies' risks of bias qualitatively. 


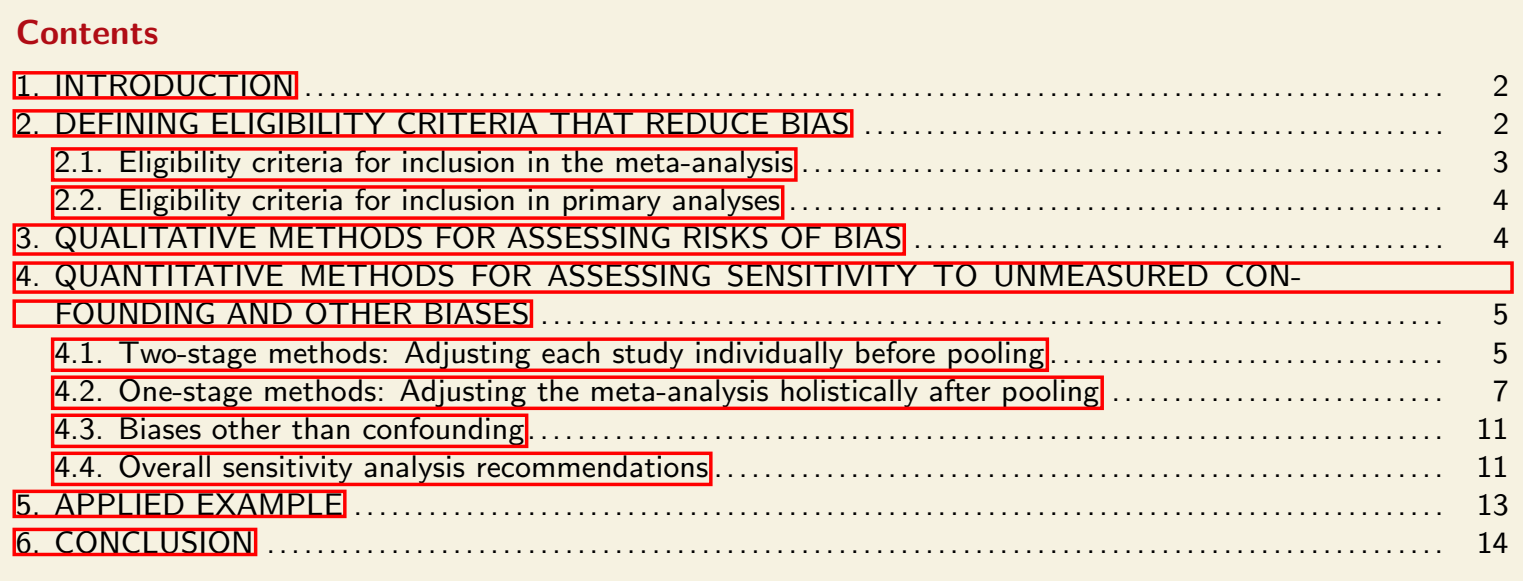

\section{INTRODUCTION}

Meta-analyses contribute critically to cumulative science (5, 14), but they can produce biased estimates and misleading conclusions if their constituent primary studies are themselves biased. Nonrandomized studies may be particularly prone to unmeasured confounding, misclassification, selection bias, and other biases. We provide practical guidance on how meta-analysts can address these biases that affect studies' internal validity, first briefly covering approaches for defining study eligibility criteria that reduce bias (Section 2) and for qualitatively assessing studies' risks of bias (Section 3). We then address our primary focus, namely methods for quantitatively assessing how sensitive meta-analysis results may be to residual bias that cannot be eliminated by limiting study eligibility (Section 4). This last topic has received relatively less attention both in the methodological literature on metaanalysis and in empirical meta-analyses (11), partly because early methods were reasonably criticized for invoking strong statistical assumptions and requiring extensive statistical expertise to implement and interpret (19). However, as we discuss, several recently developed methods have made progress on these fronts - although they do still have limitations and we therefore advocate for routinely using both qualitative and quantitative methods to assess risks of bias in individual studies and in the meta-analysis as a whole. We illustrate the use of selected quantitative sensitivity analyses in an applied example (Section 5). We focus primarily on meta-analyses whose research questions concern causation and in which the most critical bias is unmeasured confounding, although we comment on other biases throughout, especially in Section 4.3 . We do not address publication bias and similar selection processes and so refer to biases affecting studies' internal validity simply as "bias".

\section{DEFINING ELIGIBILITY CRITERIA THAT REDUCE BIAS}

We recommend that attention to reducing bias in a meta-analysis begin as early as the protocol design stage, during which the eligibility criteria for studies' inclusion in the metaanalysis and in primary versus secondary analyses can be crafted to reduce bias. Preferably, these eligibility criteria, along with the rest of the meta-analysis protocol, should be preregistered formally, with any post hoc deviations disclosed in the final manuscript (19, 37).

$2 \quad$ Mathur and VanderWeele. 


\subsection{Eligibility criteria for inclusion in the meta-analysis}

First, the meta-analyst must decide whether to include non-randomized studies (NRS) at all, and if so, whether to include only certain types of NRS. If an initial scoping review identifies a number of relevant, well-conducted randomized studies (RS) on the topic of interest, limiting eligibility to RS may provide the least biased results and still permit reasonable statistical precision. However, there may be very few (or no) relevant RS, for example because it is not feasible or ethical to randomize the exposure. Alternatively, in some contexts, RS may be available but may be subject to limitations that NRS help mitigate. For example, if the available RS use less externally generalizable samples or shorter follow-up periods than NRS, then including NRS in the meta-analysis may better address the research question (19). When including NRS in a meta-analysis, we recommend that eligibility nevertheless be restricted to study designs that provide reasonably credible evidence given the specific biases that are relevant to a given scientific topic (19); however, when few welldesigned NRS are available, deciding how stringent to be can create challenging tradeoffs between bias and precision.

Regarding confounding specifically, NRS are generally least susceptible to bias when they use longitudinal designs with the exposure measured before the outcome and when they control for confounders measured at baseline, ideally including baseline measures of the exposure and outcome themselves ${ }^{a}$ On the other hand, cross-sectional studies that measure the exposure, outcome, and any adjusted covariates contemporaneously are usually quite prone to confounding because the temporal ordering of the variables is unclear. That is, the direction(s) of causation between the exposure and outcome often cannot be established and the adjusted covariates may not permit adequate control of confounding (53, 58). For this reason, we typically recommend that cross-sectional studies be excluded altogether in meta-analyses whose research questions concern causation, except if the exposure clearly precedes the outcome despite their contemporaneous measurement (e.g., if the exposure is fixed at birth or the outcome is mortality) ${ }^{b}$ The Summary below provides a more detailed, but approximate, ranking of NRS study design features by the level of robustness to confounding that they typically provide (adapted with permission from (53)).

\footnotetext{
${ }^{a}$ Methods to control for confounders and baseline measures of the exposure and outcome include, for example, adjusting for covariates, using inverse-probability weighting, and using stratification or subset analyses. Specifically regarding the baseline outcome, another method is using withinsubject change scores as the outcome in analyses. A common form of subset analysis to control for baseline outcome values is to recruit only individuals who have not yet experienced the outcome (e.g., myocardial infarcation or mortality).

${ }^{b}$ More stringently, meta-analysts could include only cross-sectional studies in which not only (i) the exposure temporally precedes the outcome; but also (ii) the confounders temporally precede the exposure (e.g., the confounders might be age, sex, and childhood socioeconomic status, and the exposure might be adulthood socioeconomic status). In terms of robustness to confounding, these 2 criteria are preferable to criterion (i) alone because covariates measured after the exposure are not structurally confounders, and adjusting for them may not adequately control for confounding (51). However, in practice, longitudinal studies often do not report when adjusted covariates were measured, so it may be difficult to apply criterion (ii) in meta-analyses.
} 


\section{SUMMARY: A HIERARCHY OF NONRANDOMIZED DESIGNS FOR CONTROL-}

LING CONFOUNDING

In ascending order of robustness to confounding:

1. Cross-sectional data with exposure and outcome measured contemporaneously

2. Longitudinal data with exposure preceding outcome and control for baseline confounders

3. Longitudinal data with control for baseline confounders and baseline outcome

4. Longitudinal data with control for baseline confounders, outcome, and exposure

5. Longitudinal data using time-varying exposures and confounding control

If the meta-analyst is concerned about biases besides confounding, risk-of-bias tools for NRS can provide guidance on what design features could be used as inclusion criteria (48). When reviewing articles for inclusion, these design features should preferably be assessed not based on study authors' own labels for study designs (e.g., "longitudinal study"), which are defined inconsistently, but rather based on studies' actual design features, such as those in the Summary above (19). Methods to conduct literature searches for NRS are discussed elsewhere (19).

\subsection{Eligibility criteria for inclusion in primary analyses}

In meta-analyses that include both RS and NRS or that include NRS whose designs provide substantially different levels of robustness to confounding, we recommend pre-specifying in the meta-analysis protocol which designs will be included in primary and in secondary analyses (19). In general, we recommend analyzing RS and NRS separately, at least in secondary analyses (19). Regarding confounding, if the meta-analyst anticipates that the literature contains very few (if any) RS, primary analyses could be conducted using any available RS plus longitudinal NRS that measure the exposure before the outcome and that control for baseline confounders and the baseline outcome; secondary analyses could then stratify by randomization status using subset analyses or meta-regression methods (17, 19, 35, 39, 49). Similar secondary analyses could be conducted using risk-of-bias ratings, described in Section 3 Additionally, NRS often report estimates that adjust for different sets of confounders, and ideally, the meta-analysis protocol would also pre-specify which of these estimates will be extracted. In general, we recommend that the estimate that adjusts for the largest number of pre-exposure confounders be extracted for primary analyses, but when unadjusted estimates are also available, these could potentially also be extracted for secondary analyses.

\section{QUALITATIVE METHODS FOR ASSESSING RISKS OF BIAS}

We recommend that meta-analyses of NRS conduct detailed risk-of-bias (ROB) assessments on each study (19). The ROBINS-I tool provides particularly well-informed guidance on the design features that most contribute to risks of confounding and other biases (48); guidance on its use and reporting are provided elsewhere (19). We would suggest that meta-analyses of NRS report on risks of bias in at least 3 ways: (i) for the meta-analysis as a whole, the number and percent of studies occupying each level of the hierarchy given in the Summary

4 Mathur and VanderWeele. 
box above; (ii) for each study, its summary and domain-specific ROB ratings assessed using ROBINS-I; and (iii) for each study, the list of pre-exposure confounders that were adjusted in the estimate that was extracted for primary meta-analyses.

These methods for detailing each study's risks of bias and design features are integral for meta-analyses of NRS, but it can be challenging to intuit how these individual characteristics contribute to the aggregate bias in the meta-analysis results. A common method to do so, and that required in Cochrane Collaboration reviews, is the GRADE approach (19, 43). In this approach, the meta-analyst first heuristically gauges the "proportion of information" in the meta-analysis that is contributed by studies at low versus high risks of various types of bias (19, 43). Using this heuristic assessment, the meta-analyst can choose to downgrade the overall certainty rating of the meta-analysis results from the default "high certainty" to "moderate", "low", or "very low". At the meta-analyst's discretion, the certainty rating could be upgraded again if the pooled estimate is large (GRADE suggests the criterion of risk ratio $>2$ or $<0.5$ ), if there is evidence of dose-response, or if the biases are thought to have attenuated rather than inflated estimates (43).

GRADE and other qualitative approaches to assessing aggregate risks of bias are useful, but have limitations. Intuiting how much "information" each study contributes to the metaanalysis is difficult when studies' standard errors and estimates differ, and considerable subjectivity is involved in deciding how to downgrade or upgrade the overall certainty rating, as the GRADE Working Group discusses (43). Additionally, the GRADE approach ultimately provides a 4-tiered qualitative rating of the overall certainty of the results, rather than a quantitative summary of how numerical estimates might have been affected by bias. For these reasons, we encourage supplementing these qualitative methods with quantitative methods for assessing the sensitivity of meta-analysis results to bias (Section 4).

\section{QUANTITATIVE METHODS FOR ASSESSING SENSITIVITY TO UNMEASURED CONFOUNDING AND OTHER BIASES}

Sensitivity analyses are quantitative methods that characterize how numerical estimates might be affected by bias. We classify sensitivity analyses into two-stage and one-stage methods. Two-stage methods first adjust each study's point estimate and potentially also its variance, and then meta-analyze these bias-corrected estimates. In contrast, one-stage methods correct the meta-analysis holistically by specifying the distribution of bias across studies, rather than in each study individually. Below, we primarily discuss conceptually distinct methods that could be used in the context of unmeasured confounding (although many of these methods also accommodate other biases; Section 4.3) and are reasonably straightforward to implement in practice without extensive customization. Supplemental Tables 1A-1B provide additional details on the methods.

\subsection{Two-stage methods: Adjusting each study individually before pooling}

Two-stage methods begin by adjusting each individual study using any of 4 broad approaches. First, some methods use subjective elicitation, in which expert reviewers subjectively specify a numerical value for the severity of bias in each study as well as their own uncertainty in making each judgment (50). Each study's estimate is then corrected using the specified bias, and its variance estimate is inflated to accommodate subjective uncertainty $(50)$. 
Second, external adjustment methods adjust each study using information from an "external" study, which itself may or may not be included in the meta-analysis. For example, Greenland \& O'Rourke (13) proposed adjusting each meta-analyzed NRS using information from a comparably designed external study that reports both an estimate that is thought to be fully adjusted for confounding (and so is unbiased) and a partially adjusted estimate that is subject to the same amount of confounding bias as the meta-analyzed estimate to be adjusted.

Third, methods based on multiple imputation are related to external adjustment, but apply in the special context in which the meta-analyst has access to individual participant data for all studies. Under the assumption that at least some studies are fully adjusted and that, in the remaining partially adjusted studies, confounder data are missing at random, individual participants' confounder values are imputed based on relationships among the observed variables, using multilevel models to account for heterogeneity across studies in the joint distribution of the observed variables (2, 20, 40). Each partially adjusted study can then be adjusted based on these imputed confounder values using any standard method for measured confounding, such as regression adjustment or propensity score methods.

Fourth, some methods use analytical bias formulas to adjust each study given hypothetical sensitivity parameters regarding the severity and distribution of unmeasured confounder(s). For example, Goto et al.'s method (12) assumes that each study has a single, categorical unmeasured confounder; under this assumption, each study's estimate can be corrected using 4 sensitivity parameters characterizing the confounder's prevalences among the unexposed and among the exposed subjects as well as the confounder's strengths of association with the exposure and with the outcome (1) ${ }^{C}$

After obtaining bias-corrected estimates for each study using one of the above methods, two-stage methods then proceed to meta-analyze the bias-corrected estimates. Some methods simply conduct a standard meta-analysis in this second stage, without directly modeling additional uncertainty introduced by unmeasured confounding, because they essentially treat any sensitivity parameters used to obtain the corrected estimates as hypothetical fixed values (12). Other methods inflate the variances of the corrected estimates to reflect statistical error associated with the external data (13) or subjective uncertainty associated with subjective elicitation (50). In multiple imputation methods, increases in uncertainty due to unmeasured confounding are naturally captured by the between-imputation variance, which contributes to the final, pooled variance estimate (41). A conceptually unique partial identification approach first bounds each study's causal effect using bounds on the possible values of the outcome variable, then bounds the pooled estimate by taking the intersection of all the studies' bounds (27). This approach is unusual in that it provides an interval rather than a point estimate, assumes there is no effect heterogeneity across studies, and may often yield no interval at all in meta-analyses of more than a few studies.

4.1.1. Advantages and disadvantages. The key advantage of two-stage methods is that they allow case-by-case adjustment of each study based on extensive information regarding its magnitude of bias. When such information is available, is accurate, and fulfills any necessary statistical assumptions (Supplemental Table 1A), these methods can allow for accurate and precise bias correction. With sufficiently detailed data from fully adjusted studies, some

${ }^{c}$ In practice, Goto et al. (12) in fact specified the same 4 sensitivity parameters for all studies, but the method would naturally allow specification of different sensitivity parameters for each study. 
methods have the important advantage of directly and objectively correcting inference for uncertainty introduced by unmeasured confounding (2, 13, 20, 40). Additionally, there is a rich literature on methods to handle confounding and other biases in individual studies (reviewed in (23, 61) ), and in principle two-stage methods could use any of these existing methods in the first stage.

However, two-stage methods' reliance on extensive information about each study is also a disadvantage, as this information may often be unattainable for any given study, let alone when meta-analyzing many existing studies. For example, external adjustment and multiple imputation methods require extensive data from fully adjusted studies, and if these "fully adjusted" studies in fact still have residual confounding, the methods may not adjust adequately. Two-stage methods using analytical formulas require fairly detailed information and assumptions about the unmeasured confounder(s), for example that there is a single, categorical unmeasured confounder with known prevalences (12). Of the twostage methods, those using subjective elicitation require perhaps the fewest "inputs", but at the cost of relying critically on expert reviewers' ability to numerically estimate the severity of confounding bias in each study (50).

4.1.2. Software. Multiple imputation methods can be implemented using well-established $\mathrm{R}$ packages (4, 20). To our knowledge, no software is available for the other methods, but all would be straightforward to implement in any command language for statistical analysis (e.g., R or SAS) by coding a few lines of analytical bias formulas.

\subsection{One-stage methods: Adjusting the meta-analysis holistically after pooling}

One-stage methods occupy 2 broad categories: bias correction methods and E-value analog methods (Supplemental Table 1B).

4.2.1. Bias correction methods. These methods specify a hypothetical distribution of bias across studies and then obtain a bias-corrected pooled estimate or distribution of effects. McCandless et al. (36) proposed a Bayesian approach in which log- and logit-normal hyperpriors are specified on the distribution across studies of 3 sensitivity parameters: (i) the unmeasured confounder's strength of association with the outcome, conditional on the exposure and on any measured confounders; (ii) the confounder's prevalence among the unexposed group; and (iii) the confounder's prevalence among the exposed group. Assuming that, across studies, the sensitivity parameters are independent of one another and of studies' causal population effects, McCandless et al. (36) then obtained a bias-corrected likelihood and posterior for the meta-analysis by arithmetically correcting studies' estimates using these 3 sensitivity parameters. Critically, the bias formula they used to do so assumes that each study has a single, binary unmeasured confounder that does not interact with the exposure and that is independent of any measured confounders, conditional on the exposure (25). The latter assumption is highly problematic because it is in fact always violated when the measured and unmeasured confounders affect the exposure (18, 52); thus, while we do not recommend applying this method as-is, the general Bayesian approach could be adapted to use other bias formulas that obviate this assumption. Unlike two-stage methods that require the meta-analyst to specify sensitivity parameters for each study individually, the Bayesian framework requires the meta-analyst to specify only the means and variances of the sensitivity parameters' hyperpriors across studies. 
Another method considers confounding bias that is additive on the scale on which studies' estimates are meta-analyzed (e.g., the log-risk ratio scale) and that is assumed to be distributed normally across studies, again independently of studies' causal population effects (34). This method characterizes evidence strength in the meta-analysis in terms of the proportion $\left(\widehat{P}_{>q}\right)$ of causal population effects that are meaningfully strong, defined as effects above a threshold $(q)$ that the meta-analyst has chosen to represent a meaningfully strong causal effect in the scientific context (e.g., risk ratio $[R R]=1.1$ or some other threshold) (For meta-analyses with pooled estimates in the apparently preventive direction, meaningfully strong causal effects could be defined as those below a threshold, such as $R R=0.90$.) Additionally, the meta-analyst can estimate the proportion of effects below a second, possibly symmetric, threshold in the opposite direction from the pooled estimate. These proportion metrics were recently introduced in the general context of random-effects meta-analysis in order to better convey evidence strength across heterogeneous effects than the pooled estimate alone (31) e

To bias-correct the proportion of meaningfully strong causal effects, Mathur \& VanderWeele assumed that the additive bias is log-normal across studies (34). The meta-analyst would specify as sensitivity parameters the mean and variance across studies of these biases. Mathur \& VanderWeele discuss methods to choose these parameters (34); for example, the variance of the biases could be calculated by first specifying the proportion of the confounded heterogeneity estimate $\left(\widehat{\tau}_{c}^{2}\right)$ that is in fact due to heterogeneous bias. The metric $\widehat{P}_{>q}$ can then be estimated using simple arithmetic expressions involving these sensitivity parameters along with estimates from the confounded meta-analysis (34). Comparable nonparametric methods can estimate $\widehat{P}_{>q}$ without making the usual assumption in metaanalysis that the causal population effects are normal (33) or independent (35), and they provide inference that performs better in small meta-analyses or those with extreme true proportions. These methods specify a single fixed value for the bias in all studies ("homogeneous bias"). In some cases, assuming homogeneous bias yields a conservative estimate: for example, if the bias-corrected mean estimate is greater than the threshold $q$, then estimating $\widehat{P}_{>q}$ under the assumption of homogeneous bias will typically be an underestimate (representing greater sensitivity to unmeasured confounding) if in fact the bias is heterogeneous. in the Supplement, we detail the conditions under which the nonparametric estimate $\widehat{P}_{>q}$ is conservative, and we provide simple alternative expressions that are conservative under other conditions (e.g., when the bias-corrected mean estimate is less than $q$ ).

4.2.2. E-value analog methods. As described above, bias correction methods specify the severity of bias across studies to obtain a corrected pooled estimate. Conversely, E-value analog methods characterize the severity of bias that would be required, hypothetically, to shift the pooled estimate to the null or to otherwise "explain away" the results of the

\footnotetext{
${ }^{d}$ Mathur \& VanderWeele (31) discussed a number of methods to choose these thresholds, which included considering the size of discrepancies between naturally occurring groups of interest, effect sizes produced by well-evidenced interventions, cost-effectiveness analyses, or minimum subjectively perceptible thresholds.

${ }^{e}$ For example, these metrics can help identify if: (i) few effects of scientifically meaningful size exist despite a "statistically significant" pooled estimate; (ii) some large effects also exist despite an apparently null point estimate; or (iii) strong effects in the direction opposite of the pooled estimate also regularly occur (31). These metrics can also sometimes help adjudicate apparent conflicts between multiple meta-analyses (24, 30).
} 
meta-analysis. These methods are thus similar to the E-value, a recently introduced sensitivity analysis for unmeasured confounding in individual studies that does not require assumptions on the nature of unmeasured confounder(s) (8, 54). This standard E-value represents the minimum strength of association, on the $R R$ scale, that unmeasured confounder(s) would need to have with both the exposure and the outcome, conditional on any measured covariates, to fully explain away the observed exposure-outcome association in an individual study (8, 54). When the confounded estimate in an individual study, $\widehat{R R^{c}}$, is apparently causative $\left(\widehat{R R^{c}}>1\right)$, the E-value is:

$$
\text { E-value }=\widehat{R R^{c}}+\sqrt{\widehat{R R^{c}}\left(\widehat{R R^{c}}-1\right)}
$$

When instead $\widehat{R R^{c}}<1$, one would first take its inverse before applying Eq. 4.1 (8, 54). Additional details on the E-value, including how to apply and interpret it for effect measures other than $R R \mathrm{~s}$, are discussed elsewhere (8, 54, 55, 57), and reporting guidelines are provided in (57). The same considerations apply for the meta-analysis analogs discussed below. It is critical to note that the E-value and its meta-analysis analogs do not estimate the actual severity of bias, but rather describe a hypothetical severity of bias that could suffice to explain away results. Additionally, the E-value is conservative in that it considers the maximum bias that could be generated by a given strength of confounder associations, but actual unmeasured confounders might not generate that much bias (8, 54).

As a simple E-value analog for a meta-analysis, Eq. (4.1) could be directly applied to the pooled estimate transformed to the $R R$ scale (34). This E-value analog represents the average strengths of association across studies, on the $R R$ scale, that unmeasured confounder(s) would need to have with studies' exposures and outcomes in order to shift the pooled estimate to the null. Additionally, one can consider the severity of confounding that would be required to shift the confidence interval for the pooled estimate to include the null; to do so, $\widehat{R R^{c}}$ in the above expression would simply be replaced with the confidence interval limit closer to the null (34, 54). These metrics do not make assumptions on the distribution of the bias in the confounded population effects, although again, the bias must be independent of studies' standard errors (Supplement).

Like most sensitivity analyses for meta-analyses, this simple E-value analog is limited to characterizing evidence strength only in terms of the pooled estimate and its confidence interval. Other E-value analogs instead characterize evidence strength in terms of the aforementioned proportion of meaningfully strong causal effects, $\widehat{P}_{>q}$ (34). For example, Mathur \& VanderWeele (34) proposed a metric, $\widehat{G}(r, q)$, that represents the minimum average strengths of association on the $R R$ scale that unmeasured confounder(s) would need to have with both the exposure and the outcome in order to reduce to less than some value $r$ (e.g., 0.15) the proportion of studies with causal population effects stronger than $q$. The rationale for this approach is that, when effects are heterogeneous, one might define "explaining away" the results of the meta-analysis in terms of substantially reducing the proportion of meaningfully strong effects in this way. Letting $\widehat{\mu}^{c}$ and $\widehat{\tau}_{c}^{2}$ denote the pooled estimate and heterogeneity estimate from the confounded meta-analysis, $\sigma_{B^{*}}^{2}$ denote the across-study variance of the log-normal bias, and $\Phi$ denote the normal cumulative distribution function, the metric $\widehat{G}(r, q)$ can be estimated as follows for a confounded pooled 
estimate that is apparently causative $\left(\widehat{\mu}^{c}>0\right.$ on the log- $R R$ scale)

$$
\begin{gathered}
\widehat{G}(r, q)=\widehat{T}(r, q)+\sqrt{(\widehat{T}(r, q))^{2}-\widehat{T}(r, q)} \\
\widehat{\mathrm{SE}}(\widehat{G}(r, q))=\widehat{\mathrm{SE}}(\widehat{T}(r, q)) \cdot\left(1+\frac{2 \widehat{T}(r, q)-1}{2 \sqrt{\widehat{T}(r, q)^{2}-\widehat{T}(r, q)}}\right)
\end{gathered}
$$

where

$$
\begin{gathered}
\widehat{T}(r, q)=\exp \left\{\Phi^{-1}(1-r) \sqrt{\widehat{\tau}_{c}^{2}-\sigma_{B^{*}}^{2}}-q+\widehat{\mu}^{c}\right\} \\
\widehat{\mathrm{SE}}(\widehat{T}(r, q))=\exp \left\{\left(\Phi^{-1}(1-r)\right) \sqrt{\widehat{\tau}_{c}^{2}-\sigma_{B^{*}}^{2}}-q+\widehat{\mu}^{c}\right\} \sqrt{\widehat{\operatorname{Var}}\left(\widehat{\mu}^{c}\right)+\frac{\widehat{\operatorname{Var}}\left(\widehat{\tau}_{c}^{2}\right)\left(\Phi^{-1}(1-r)\right)^{2}}{4\left(\widehat{\tau}_{c}^{2}-\sigma_{B^{*}}^{2}\right)}}
\end{gathered}
$$

(The intermediate estimate $\widehat{T}(r, q)$ represents multiplicative bias on the $R R$ scale regardless of its origin, discussed further in Section 4.3) Similar expressions for the apparently preventive case, $\widehat{\mu}^{c}<0$, appear in the Supplement.

As for $\widehat{P}_{>q}$, comparable nonparametric methods $(33,35)$ can estimate $\widehat{G}(r, q)$ in a wider range of settings than is possible using the above parametric methods. The nonparametric methods assume homogeneous bias across studies, but can sometimes be interpreted as a conservative estimate (Supplement).

4.2.3. Advantages and disadvantages. In contrast to two-stage methods that require extensive information and assumptions about the severity of bias in each study, one-stage bias correction methods require specification of only a small number of sensitivity parameters that characterize the severity of bias across all studies. E-value analog methods require yet fewer, if any, sensitivity parameters to be specified because, conversely to bias correction methods, E-value methods solve for the severity of bias that would have to exist in order to explain away the results of the meta-analysis. This has the advantage of reducing "researcher degrees of freedom" associated with choosing sensitivity parameters post hoc, which might be especially problematic with two-stage methods (44) and with qualitative evidence-grading systems (43). Whereas all two-stage methods require access to at least study-level estimates and variances (Supplemental Table 1A), often precluding analysis by third parties, certain one-stage methods can be conducted using only statistical estimates from the meta-analysis itself, allowing for sensitivity analysis of some published meta-analyses for which study-level data are unavailable (34, 54).

However, by eliminating case-by-case specification of bias parameters, one-stage methods typically introduce assumptions about the distribution of bias across studies that are unnecessary for most two-stage methods. Most one-stage methods either assume that sensitivity parameters are homogeneous across studies or that they are are log- or logit-normal. Diagnostic plots and tests can sometimes be used to rule out severe violations of these assumptions; for example, the assumptions of Mathur \& VanderWeele (34) imply that the population confounded effects are normal, so standard normality tests for meta-analyses

\footnotetext{
${ }^{f}$ These expressions are straightforward generalizations of those given in (34). That paper had defined $\widehat{T}(r, q)$ and $\widehat{G}(r, q)$ for the case of homogeneous bias $\left(\sigma_{B^{*}}^{2}=0\right)$; here we give expressions that accommodate heterogeneous bias. 
could be used (16, 59). Nonparametric methods (33) can sometimes be calculated and interpreted under weakened assumptions on the bias distribution (Supplement). Also, most one-stage methods make the important assumption that the bias in each study is independent of its population causal effect 9 which could be violated if, for example, study authors who investigate small causal effects tend to adjust for only a few confounders in order to obtain "statistically significant" results. We give some practical guidance for navigating statistical assumptions in Section 4.4

4.2.4. Software. McCandless et al.'s (36) method could be implemented by modifying their example R code; as noted in Section 4.2 we consider it critical to use a different bias formula when applying the general Bayesian framework. All other one-stage methods discussed above (33, 34) can be implemented using the website http://www.evalue-calculator. com/meta/, or the R package EValue (28), for which vignettes are available (29). We provide a step-by-step tutorial for using this website and R package in the Supplement.

\subsection{Biases other than confounding}

Although we have focused on methods that can provide sensitivity analyses at least for unmeasured confounding, some of these methods also naturally accommodate other biases in NRS or RS, such as participant selection, measurement error, missing data, or noncompliance (Supplemental Tables 1A-1B). For example, in principle, meta-analysts could subjectively elicit any type of bias (50). Bayesian methods have been proposed for other biases (47, 60). Methods to handle psychometric artifacts arising from, for example, range restriction of the outcome or imperfect construct validity are detailed elsewhere (42).

One-stage sensitivity analyses for unmeasured confounding (33, 34) could be readily adapted for certain other biases for which expressions equivalent to the E-value are now available for individual studies (selection bias (46), differential measurement error (56), and combinations of these biases with unmeasured confounding (45)). These E-value equivalents represent the severity of bias, in terms of sensitivity parameters that are specific to the bias under consideration, that would be required shift the effect of an individual study to the null. To apply these results for a meta-analysis, the meta-analyst could first estimate $\widehat{T}(r, q)$, which represents multiplicative bias on the $R R$ scale regardless of origin, exactly as described above (33, 34), and then could calculate a bias-specific analog to $\widehat{G}(r, q)$ by transforming $\widehat{T}(r, q)$ using the relevant bias-specific "E-value" expression (45, 46, 56).

\subsection{Overall sensitivity analysis recommendations}

We recommend that meta-analyses of NRS routinely report one or more sensitivity analyses for unmeasured confounding and potentially for other biases as relevant to the scientific context and study designs. This recommendation in no way detracts from the importance of also implementing the recommendations in Sections 23 As noted above, all sensitivity analysis methods make statistical assumptions of varying stringency, and many sensitivity analyses require extensive information characterizing the amount of bias in each study. Additionally, many methods are not yet implemented in software.

\footnotetext{
${ }^{g}$ However, these methods do not assume that the bias is independent of the confounded estimates: naturally, studies with more severe bias may tend to estimate systematically larger or smaller effect sizes.
} 
Given these considerations, one possible practical approach for choosing among the methods is as follows. First, the meta-analyst could calculate and report the following simple E-value analogs: (i) the E-value for the pooled estimate and its confidence interval limit closer to the null, which respectively represent the average severity of confounding across studies that would be required to shift the pooled estimate, and to shift its confidence interval, to the null (34, 54); and (ii) a nonparametric estimate of $\widehat{G}(r, q)$, which represents the severity of homogeneous confounding that would need to be present in each study in order to reduce to less than $r$ the proportion of causal population effects stronger than a chosen threshold, $q$ (33, 34). As discussed in Section 4.2 and the Supplement, the metric $\widehat{G}(r, q)$ is perhaps most informative when it is calculated and interpreted under conservative assumptions, rather than under the strict assumption that the bias truly is homogeneous.

We believe that, as a generic starting point, reporting these simple metrics is reasonable because these metrics apply to a fairly broad range of meta-analyses of NRS: (i) they do not make assumptions about the nature of unmeasured confounder(s) themselves within studies (e.g., the metrics accommodate multiple confounders, non-binary confounders, and confounders that interact with the exposure); (ii) they require no specification of sensitivity parameters; and (iii) they are straightforward to implement using standard study-level data and available software (28, 29). Additionally, these metrics characterize the sensitivity to unmeasured confounding of both the pooled estimate (and its confidence interval) and the proportion of meaningfully strong causal effects; they thus provide a straightforward way to summarize the distribution of causal effects in the meta-analysis in terms of both its mean and its variability. (If the heterogeneity estimate $\widehat{\tau}_{c}^{2}$ is 0 , then the metric $\widehat{G}(r, q)$ would be omitted.) As such, the metrics provide complementary information: depending on the distribution of population effects, the point estimate may be more or less sensitive to confounding than the percentage of meaningfully strong effects.

Given the results of these simple sensitivity analyses, we recommend that the metaanalyst then attempt to assess and report whether it is actually plausible that the metaanalyzed studies are subject to confounding as severe as that indicated by the E-values and by $\widehat{G}(r, q)$. Examples can be found in existing meta-analyses (3, 10, 26). This assessment would be based on substantive knowledge of the exposures and outcomes under consideration and the ROB assessments described in Section 2 For example, more unmeasured confounding would be plausible in a meta-analysis of cross-sectional studies than in an otherwise comparable meta-analysis of longitudinal studies that control for an ample set of baseline confounders, including baseline values of the exposure and outcome (53, 58). Additionally, examining the confounding associations of measured confounders with the exposure and outcome, for example from studies that report both adjusted and unadjusted estimates, can also help inform assessments of the plausible severity of unmeasured confounding. However, even if measured confounders have strong confounding associations, residual unmeasured confounding above and beyond these strong measured confounders may be considerably less severe. Also, because the E-value considers maximum bias, even if unmeasured confounders do in fact have confounding associations similar in magnitude to the E-value, this does not necessarily mean that these confounders could actually explain away the effect, only that the evidence is less clear. Last, some empirical studies have more broadly assessed the extent of agreement or disagreement between NRS and RS on the same topic (e.g., those included in the same meta-analysis); we summarize several such results in the Supplement. However, it is critical to note that disagreements between NRS and RS cannot be interpreted as direct estimates of confounding bias, but rather of the ag- 
gregation of confounding bias plus any other systematic differences between study designs. Furthermore, the severity of confounding differs across meta-analyses and scientific topics.

In general, we would advise also conducting sensitivity analyses that consider more precise forms of heterogeneous bias across studies. One possible approach is to apply onestage methods that assume log-normal bias across studies and do not make assumptions about the nature of confounder(s) within each study; as discussed above, these methods also characterize the heterogeneous distribution of population effects (34). If the metaanalyst is concerned about a specific, single unmeasured confounder with known prevalences, one-stage Bayesian methods that similarly assume log-normal and logit-normal sensitivity parameters across studies could be adapted (36), again replacing the existing bias formula with one that obviates the problematic conditional independence assumption. If the metaanalyst has access to the specific forms of external data or individual participant data required by two-stage methods (Supplemental Table 1A), then these methods could be applied to obviate distributional assumptions on the bias and to provide potentially more accurate bias-corrected estimates, albeit by introducing different statistical assumptions.

\section{SUMMARY: SENSITIVITY ANALYSIS RECOMMENDATIONS}

1. When meta-analyzing NRS, report sensitivity analyses for unmeasured confounding and potentially other biases, even when following the principles in Sections 23

2. As a starting point, consider reporting (i) the E-value for the pooled estimate and its confidence interval; and (ii) the amount of homogeneous confounding, $\widehat{G}(r, q)$, that would be required to substantially reduce the proportion of meaningfully strong causal effects.

3. Consider also conducting further one-stage or two-stage sensitivity analyses that accommodate more precisely specified forms of heterogeneous bias.

4. Interpret and report the results of these sensitivity analyses in the context of studies' risks of bias.

\section{APPLIED EXAMPLE}

We now illustrate the use and interpretation of selected one-stage sensitivity analyses by applying them to a published meta-analysis. Kodama et al. (22) meta-analyzed longitudinal studies that assessed the association of lower versus higher maximal aerobic capacity with all-cause mortality (Supplemental Figure 1). Prior to correction for unmeasured confounding, our replication of their meta-analysis using 16 studies yielded a pooled $R R$ of 1.73 (95\% confidence interval [CI]: [1.50, 1.99]; $p<0.001$; heterogeneity $\widehat{\tau}^{c}=0.20$ ). All studies were longitudinal and adjusted for some, but not all, possible confounders. The aerobic capacity measure was arithmetically adjusted for average age and sex differences, but many studies did not adjust for other possible confounders, such as smoking, body mass index, physical activity, and underlying diseases. Kodama et al. (22) did not report on whether each study measured confounders at baseline and did not rate studies' risks of bias.

We first conducted the simple sensitivity analyses described in Section 4.4 (The Supplement provides a step-by-step tutorial on how to conduct all analyses described below.) Computing the E-value for the pooled estimate indicated that unmeasured confounder(s) 
associated with both lower aerobic capacity and higher all-cause mortality by average risk ratios of 2.85-fold each could potentially shift the pooled estimate to the null; average confounding associations of 2.36-fold each could potentially shift the confidence interval to the null. To characterize the heterogeneous distribution of causal effects, we considered effects larger than $R R=1.1$ to represent meaningfully strong detrimental effects of lower aerobic capacity. We therefore chose $q=\log (1.1)$ because we conducted analyses on the $\log R R$ scale. Prior to correction for unmeasured confounding, we estimated that the percentage of studies with meaningfully strong population effects $(R R>1.1)$ was nearly $100 \%$.

We then estimated that to reduce this percentage to $15 \%$, homogeneous unmeasured confounding $R R$ s with both lower aerobic capacity and higher all-cause mortality of $\widehat{G}(r=$ $0.15, q=\log (1.1))=3.07$ (95\% CI: $[2.35,4.28])$ each could suffice, but weaker homogeneous confounding could not (Supplemental Figure 2A) (33). Although control of confounding in the meta-analyzed studies was quite limited, these sensitivity analyses seem to suggest reasonably robust evidence for effects of aerobic capacity on mortality: it seems somewhat implausible that, above and beyond measured confounding, each study had sufficiently severe unmeasured confounding (e.g., associations of $R R=2.36$ to 3.07 with both aerobic capacity and mortality) to shift the pooled estimate or its confidence interval to null, or to reduce the percentage of meaningfully strong effects to only $15 \%$.

To supplement these simple sensitivity analyses, we also assessed the sensitivity of these results to unmeasured confounding under the assumption that bias was highly heterogeneous across studies such that it accounted for $80 \%$ of the estimated total between-study variance (Supplemental Figure 2B) (34). We estimated that, to reduce the percentage of meaningfully strong causal effects to $15 \%$, unmeasured confounding $R R \mathrm{~s}$ with both higher aerobic capacity and lower all-cause mortality of on average $\widehat{G}(r=0.15, q=\log (1.1))=2.83$ (95\% CI: $[1.67,4])$ across studies would suffice to explain away the meta-analysis results in this sense, but weaker confounding would not (34). Again, this severity of unmeasured confounding seems somewhat implausible in these longitudinal studies, and thus the conclusion that there is a considerable percentage of studies with meaningfully large effects seems fairly robust to even substantial degrees of heterogeneous unmeasured confounding. This final analysis assumes that the bias was log-normal across studies; diagnostic plots did not suggest any severe violation of this assumption.

\section{CONCLUSION}

Our overall recommendations have been as follows:

\section{SUMMARY: OVERALL RECOMMENDATIONS}

1. Pre-specify study eligibility criteria that reduce risks of bias. Meta-analyses addressing causal questions should usually exclude cross-sectional studies unless the exposure clearly precedes the outcome temporally.

2. Pre-specify which study designs will be included in primary and in secondary analyses. Stratify on designs that provide substantially differing levels of evidence, at least in secondary analyses.

3. Qualitatively characterize risks of bias in terms of, at minimum: (i) the number and percent of studies occupying each level of robustness to confounding; (ii) for 
each study, its summary and domain-specific ROB ratings using ROBINS-I (48); and (iii) for each study, the list of pre-exposure confounders that were adjusted.

4. Quantitatively assess sensitivity to residual biases and interpret the results in light of the qualitative risk-of-bias assessments.

We have recommended routinely applying quantitative sensitivity analyses on the grounds that they provide informative, relatively objective quantitative summaries of evidence strength that complement more widespread qualitative approaches (Section 3). This recommendation may prove controversial: others have reasonably argued that sensitivity analysis methods require unrealistic statistical assumptions and are difficult for nonstatisticians to implement and interpret (19). However, as we have discussed, more recently developed sensitivity analyses relax some - though certainly not all - important assumptions and are straightforward to implement and interpret. We therefore believe that these methods make progress toward resolving these concerns and that the methods, when reported responsibly, can contribute substantially to characterizing the credibility of a meta-analysis.

To further advance this field, several future directions seem particularly impactful. First, it would be valuable to continue extending quantitative methods, for example to more flexibly model the propagation of uncertainty in sensitivity parameters to meta-analysis results, to characterize evidence strength using metrics that summarize heterogeneous effect distributions rather than only the pooled estimate, and to further accommodate heterogeneous bias, especially bias that is correlated with the causal population effects. Second, because interpreting sensitivity analyses requires assessing the severity of bias that is plausible in the meta-analyzed studies, it would be valuable to continue establishing empirical benchmarks for the actual severity of bias in studies on different topics and of different designs. We have reviewed some such work in the Supplement, but we particularly encourage further developments that more rigorously parse genuine bias from other systematic differences between study designs (6, 7).

Third, making datasets publicly available for both original research (with appropriate deidentification) and meta-analyses would resolve a critical and largely unnecessary limiting factor on meta-analysts' ability to handle bias. If individual participant data were routinely available, much more sophisticated quantitative methods with fewer assumptions could be developed. Meta-analysts themselves often do not make even study-level data available publicly or on request (32, 38), largely preventing third parties from conducting sensitivity analyses except sometimes by a single method (34). Simple policies and incentives by journals can sometimes rapidly improve data availability when ethical (15, 21), with many collateral benefits for the credibility and efficiency of both original research and meta-analyses.

We hope that the methods and recommendations discussed in this review, along with the suggested future directions, will help inform a balanced and nuanced view of the credibility of meta-analyses.

\section{REPRODUCIBILITY}

All data, materials, and code required to reproduce the applied example (Section 5) and the re-analysis of empirical benchmarking data (Supplement) are publicly available and 
documented (https://osf.io/yd3ws/).

\section{FUNDING}

This research was supported by (1) NIH grants R01 LM013866R01 and R01 CA222147;

(2) the NIH-funded Biostatistics, Epidemiology and Research Design (BERD) Shared Resource of Stanford University's Clinical and Translational Education and Research (UL1TR003142); (3) the Biostatistics Shared Resource (BSR) of the NIH-funded Stanford Cancer Institute (P30CA124435); and (4) the Quantitative Sciences Unit through the Stanford Diabetes Research Center (P30DK116074).

\section{ACKNOWLEDGMENTS}

We thank Sebastian Schneeweiss and the other RCT-DUPLICATE authors (9) for providing study-level data for re-analysis (Supplement).

16 Mathur and VanderWeele. 


\section{LITERATURE CITED}

1. Arah OA, Chiba Y, Greenland S. 2008. Bias formulas for external adjustment and sensitivity analysis of unmeasured confounders. Annals of Epidemiology 18:637-646

2. Audigier V, White IR, Jolani S, Debray TP, Quartagno M, et al. 2018. Multiple imputation for multilevel data with continuous and binary variables. Statistical Science 33:160-183

3. Baumeister SE, Leitzmann MF, Linseisen J, Schlesinger S. 2019. Physical activity and the risk of liver cancer: a systematic review and meta-analysis of prospective studies and a bias analysis. JNCI: Journal of the National Cancer Institute 111:1142-1151

4. Buuren Sv, Groothuis-Oudshoorn K. 2010. mice: Multivariate imputation by chained equations in R. Journal of Statistical Software :1-68

5. Cumming G. 2014. The new statistics: Why and how. Psychological Science 25:7-29

6. Dahabreh IJ, Petito LC, Robertson SE, Hernán MA, Steingrimsson JA. 2020. Toward causally interpretable meta-analysis: Transporting inferences from multiple randomized trials to a new target population. Epidemiology 31:334-344

7. Dahabreh IJ, Robins JM, Hernán MA. 2020. Benchmarking observational methods by comparing randomized trials and their emulations. Epidemiology 31:614-619

8. Ding P, VanderWeele TJ. 2016. Sensitivity analysis without assumptions. Epidemiology (Cambridge, Mass.) 27:368

9. Franklin JM, Patorno E, Desai RJ, Glynn RJ, Martin D, et al. 2020. Emulating randomized clinical trials with nonrandomized real-world evidence studies: First results from the RCT DUPLICATE initiative. Circulation

10. Fu R, Sekercioglu N, Mathur MB, Couban R, Coyte PC. 2020. Dialysis initiation and all-cause mortality among incident adult patients with advanced ckd: A meta-analysis with bias analysis. Kidney Medicine

11. Golder S, Loke YK, Bland M. 2011. Meta-analyses of adverse effects data derived from randomised controlled trials as compared to observational studies: Methodological overview. PLoS Medicine 8:e1001026

12. Goto A, Arah OA, Goto M, Terauchi Y, Noda M. 2013. Severe hypoglycaemia and cardiovascular disease: systematic review and meta-analysis with bias analysis. BMJ 347

13. Greenland S, O'Rourke K. 2008. Meta-analysis. In Modern epidemiology. Lippincott Williams \&amp; Wilkins, 3rd ed.

14. Gurevitch J, Koricheva J, Nakagawa S, Stewart G. 2018. Meta-analysis and the science of research synthesis. Nature 555:175-182

15. Hardwicke TE, Mathur MB, MacDonald K, Nilsonne G, Banks GC, et al. 2018. Data availability, reusability, and analytic reproducibility: Evaluating the impact of a mandatory open data policy at the journal Cognition. Royal Society Open Science 5:180448

16. Hardy RJ, Thompson SG. 1998. Detecting and describing heterogeneity in meta-analysis. Statistics in Medicine 17:841-856

17. Hedges LV, Tipton E, Johnson MC. 2010. Robust variance estimation in meta-regression with dependent effect size estimates. Research Synthesis Methods 1:39-65

18. Hernan M, Robins J. 1999. Letter to the editor of Biometrics. Biometrics 55:1316-1316

19. Higgins JP, Thomas J, Chandler J, Cumpston M, Li T, et al. 2019. Cochrane handbook for systematic reviews of interventions. John Wiley \&amp; Sons

20. Jolani S, Debray TP, Koffijberg H, van Buuren S, Moons KG. 2015. Imputation of systematically missing predictors in an individual participant data meta-analysis: a generalized approach using mice. Statistics in Medicine 34:1841-1863

21. Kidwell MC, Lazarević LB, Baranski E, Hardwicke TE, Piechowski S, et al. 2016. Badges to acknowledge open practices: A simple, low-cost, effective method for increasing transparency. PLoS Biology 14:e1002456

22. Kodama S, Saito K, Tanaka S, Maki M, Yachi Y, et al. 2009. Cardiorespiratory fitness as a quantitative predictor of all-cause mortality and cardiovascular events in healthy men and 
women: A meta-analysis. Journal of the American Medical Association 301:2024-2035

23. Lash TL. 2021. Bias analysis. In Modern epidemiology. Wolters Kluwer, 4th ed.

24. Lewis M, Mathur MB, VanderWeele TJ, Frank MC. 2020. The puzzling relationship between multi-lab replications and meta-analyses of the published literature Preprint: https://psyarxiv.com/pbrdk/

25. Lin DY, Psaty BM, Kronmal RA. 1998. Assessing the sensitivity of regression results to unmeasured confounders in observational studies. Biometrics :948-963

26. Ling S, Brown K, Miksza JK, Howells L, Morrison A, et al. 2020. Association of type 2 diabetes with cancer: A meta-analysis with bias analysis for unmeasured confounding in 151 cohorts comprising 32 million people. Diabetes Care 43:2313-2322

27. Manski CF. 2020. Toward credible patient-centered meta-analysis. Epidemiology 31:345-352

28. Mathur MB, Ding P, Riddell CA, VanderWeele TJ. 2018. Web site and R package for computing E-values. Epidemiology 29:e45-e47

29. Mathur MB, Ding P, VanderWeele TJ. 2020. EValue 4.1.1: Sensitivity analyses for unmeasured confounding in observational studies and meta-analyses. Https://cran.rproject.org/web/packages/EValue/

30. Mathur MB, VanderWeele TJ. 2019. Finding common ground in meta-analysis "wars" on violent video games. Perspectives on Psychological Science 14:705-708

31. Mathur MB, VanderWeele TJ. 2019. New metrics for meta-analyses of heterogeneous effects. Statistics in Medicine 38:1336-1342

32. Mathur MB, VanderWeele TJ. 2020. Estimating publication bias in meta-analyses of peerreviewed studies: A meta-meta-analysis across disciplines and journal tiers. Research Synthesis Methods

33. Mathur MB, VanderWeele TJ. 2020. Robust metrics and sensitivity analyses for meta-analyses of heterogeneous effects. Epidemiology 31:356-358

34. Mathur MB, VanderWeele TJ. 2020. Sensitivity analysis for unmeasured confounding in metaanalyses. Journal of the American Statistical Association 115:163-172

35. Mathur MB, VanderWeele TJ. 2021. Meta-regression methods to characterize evidence strength using meaningful-effect percentages conditional on study characteristics. Research Synthesis Methods Preprint retrieved from https://osf.io/bmtdq/

36. McCandless LC. 2012. Meta-analysis of observational studies with unmeasured confounders. The International Journal of Biostatistics 8:1

37. Moher D, Shamseer L, Clarke M, Ghersi D, Liberati A, et al. 2015. Preferred reporting items for systematic review and meta-analysis protocols (PRISMA-P) 2015 statement. Systematic Reviews 4:1-9

38. Polanin JR, Hennessy EA, Tsuji S. 2020. Transparency and reproducibility of meta-analyses in psychology: A meta-review. Perspectives on Psychological Science 15:1026-1041

39. Pustejovsky JE, Tipton E. 2021. Meta-analysis with robust variance estimation: Expanding the range of working models. Prevention Science :1-14

40. Resche-Rigon M, White IR, Bartlett JW, Peters SA, Thompson SG, Group PIS. 2013. Multiple imputation for handling systematically missing confounders in meta-analysis of individual participant data. Statistics in Medicine 32:4890-4905

41. Rubin DB. 2004. Multiple imputation for nonresponse in surveys, vol. 81. John Wiley \&amp; Sons

42. Schmidt FL, Hunter JE. 2015. Methods of meta-analysis: Correcting error and bias in research findings. Sage, 3rd ed.

43. Schünemann HJ, Cuello C, Akl EA, Mustafa RA, Meerpohl JJ, et al. 2019. Grade guidelines: 18. how robins-i and other tools to assess risk of bias in nonrandomized studies should be used to rate the certainty of a body of evidence. Journal of Clinical Epidemiology 111:105-114

44. Simmons JP, Nelson LD, Simonsohn U. 2011. False-positive psychology: Undisclosed flexibility in data collection and analysis allows presenting anything as significant. Psychological Science 
22:1359-1366

45. Smith LH, Mathur MB, VanderWeele TJ. In press. Multiple-bias sensitivity analysis using bounds. Epidemiology Preprint: https://arxiv.org/abs/2005.02908/

46. Smith LH, VanderWeele TJ. 2019. Bounding bias due to selection. Epidemiology 30:509

47. Spiegelhalter DJ, Best NG. 2003. Bayesian approaches to multiple sources of evidence and uncertainty in complex cost-effectiveness modelling. Statistics in Medicine 22:3687-3709

48. Sterne JA, Hernán MA, Reeves BC, Savović J, Berkman ND, et al. 2016. ROBINS-I: a tool for assessing risk of bias in non-randomised studies of interventions. BMJ 355

49. Thompson SG, Higgins JP. 2002. How should meta-regression analyses be undertaken and interpreted? Statistics in Medicine 21:1559-1573

50. Turner RM, Spiegelhalter DJ, Smith GC, Thompson SG. 2009. Bias modelling in evidence synthesis. Journal of the Royal Statistical Society: Series A (Statistics in Society) 172:21-47

51. VanderWeele T. 2015. Explanation in causal inference: methods for mediation and interaction. Oxford University Press

52. VanderWeele TJ. 2008. Sensitivity analysis: distributional assumptions and confounding assumptions. Biometrics 64:645-649

53. VanderWeele TJ. 2021. Causal inference with time-varying exposures. In Modern epidemiology. Wolters Kluwer, 4th ed.

54. VanderWeele TJ, Ding P. 2017. Sensitivity analysis in observational research: introducing the E-value. Annals of Internal Medicine 167:268-274

55. VanderWeele TJ, Ding P, Mathur M. 2019. Technical considerations in the use of the e-value. Journal of Causal Inference 7

56. VanderWeele TJ, Li Y. 2019. Simple sensitivity analysis for differential measurement error. American Journal of Epidemiology 188:1823-1829

57. VanderWeele TJ, Mathur MB. 2020. Commentary: developing best-practice guidelines for the reporting of e-values. International Journal of Epidemiology 49:1495-1497

58. VanderWeele TJ, Mathur MB, Chen Y, et al. 2020. Outcome-wide longitudinal designs for causal inference: A new template for empirical studies. Statistical Science 35:437-466

59. Wang CC, Lee WC. 2020. Evaluation of the normality assumption in meta-analyses. American Journal of Epidemiology 189:235-242

60. Wolpert RL, Mengersen KL, et al. 2004. Adjusted likelihoods for synthesizing empirical evidence from studies that differ in quality and design: effects of environmental tobacco smoke. Statistical Science 19:450-471

61. Zhang X, Stamey JD, Mathur MB. 2020. Assessing the impact of unmeasured confounders for credible and reliable real-world evidence. Pharmacoepidemiology and Drug Safety 29:1219-1227 


\title{
Supplement: Methods to Address Confounding and Other Biases in Meta-Analyses
}

\author{
Contents
}

1 Supplemental Tables 1A-1B: Summary of two-stage and one-stage methods 2

2 Using the website and R package to analyze the applied example 4

2.1 Calculating an E-value for the point estimate and its confidence interval . . . . . . . . 4

2.2 Estimating the percentage of effects above $R R=1.1$ and the strength of homogeneous confounding required to reduce the percentage to less than $15 \%$. . . . . . . . . . . 5

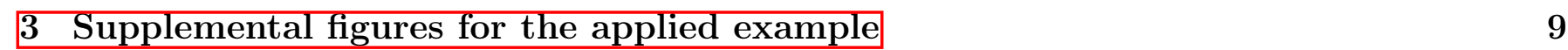

4 Empirical benchmarks on agreement between nonrandomized and randomized studies in meta-analyses

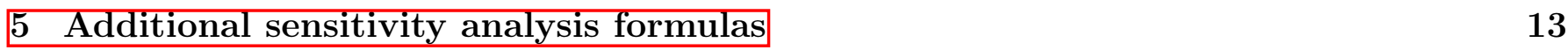

$5.1 \quad \widehat{T}(r, q)$ and $\widehat{G}(r, q)$ with log-normal bias . . . . . . . . . . . . . . . . . . . . 13

5.2 Sensitivity analysis with weakened assumptions on the bias distribution . . . . . . . . 13

$5.2 .1 \quad$ E-value for the pooled estimate and confidence interval . . . . . . . . . . . . . 13

$5.2 .2 \widehat{P}_{>q}, \widehat{G}(r, q)$, and $\widehat{T}(r, q) \ldots \ldots \ldots \ldots \ldots \ldots$ 


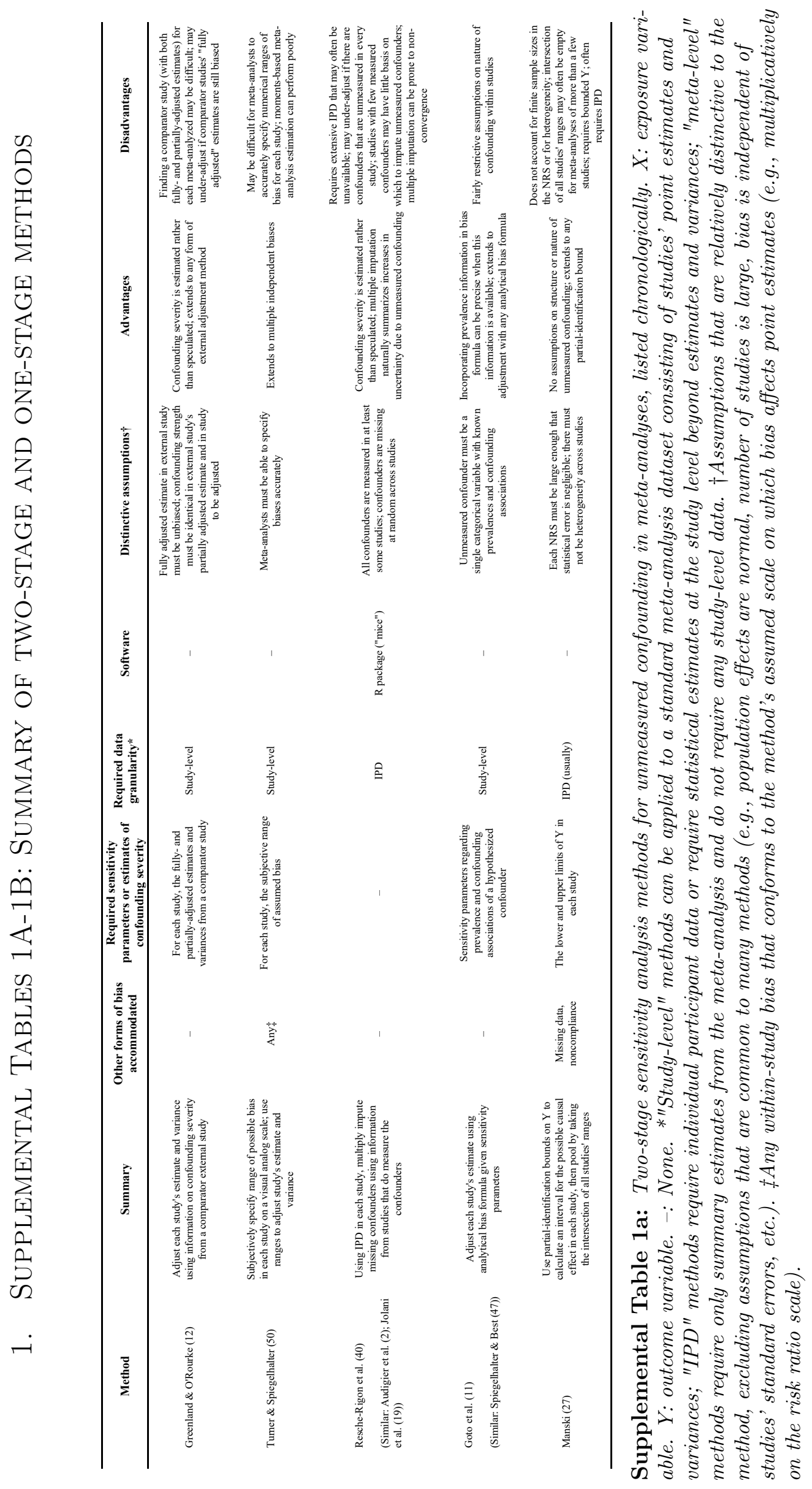




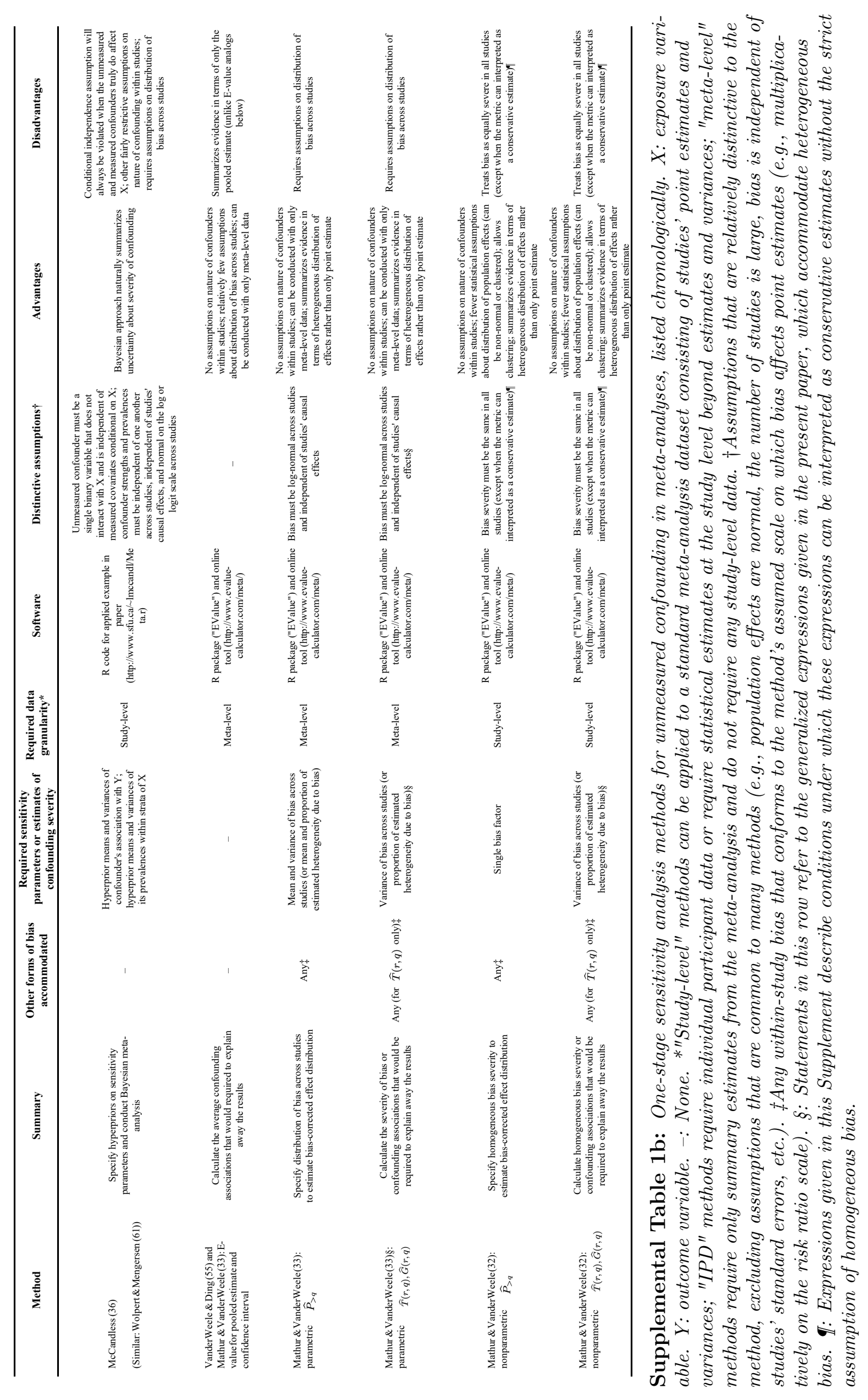




\section{Using the WeBsite And R PACKAGE to ANALyze the APPliEd EXAMPle}

Here we provide a tutorial on how to use the R package EValue (version 4.1.2) and website (www . evalue-calculator.com/meta) to re-analyze Kodama et al. (2009)'s meta-analysis on aerobic capacity and mortality, as described in the main text.

\subsection{Calculating an E-value for the point estimate and its confidence interval}

To calculate E-values, we first fit a standard random-effects meta-analysis ${ }^{\text {to }}$ to obtain the meta-analysis point estimate and confidence interval. Doing so in $\mathrm{R}$ yields the following output, with all estimates given on the log-relative risk scale (Viechtbauer et al., 2010):

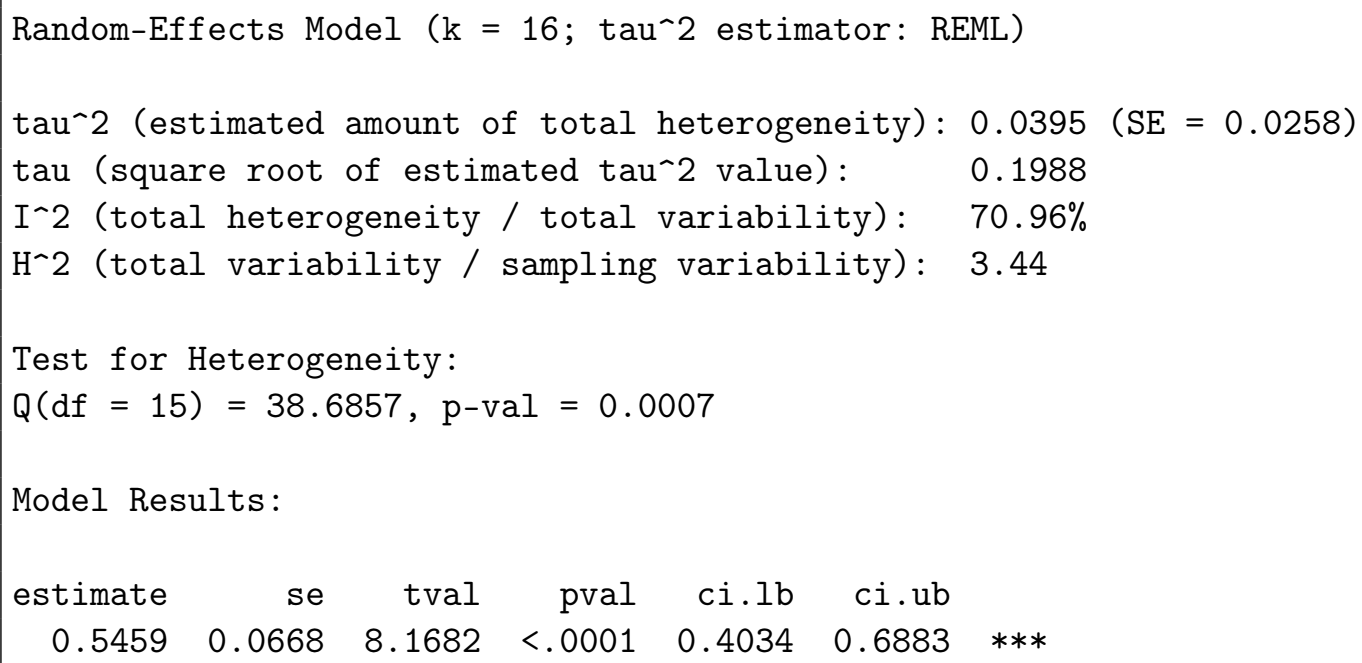

Then, in the first tab of the website www.evalue-calculator.com/meta, called "Sensitivity analysis for the point estimate", we input the pooled point estimate and confidence interval limits on the relative risk scale. We can obtain these by exponentiating the log-relative risks in the final line of the $\mathrm{R}$ output above, yielding a pooled point estimate of $\exp \{0.5459\} \approx 1.73$ with confidence interval bounds of $\exp \{0.4034\} \approx 1.50$ and $\exp \{0.6883\} \approx 1.99$. By default, the website considers shifting the point estimate and lower confidence interval limit to the null, which is usually taken to be 1 for ratio measures (but this default can be modified).

\footnotetext{
${ }^{a}$ In this meta-analysis, some papers or cohorts contributed multiple point estimates. We use "studies" to refer to the meta-analyzed point estimates. For illustrative purposes and to more closely reproduce the meta-analysts' reported results, we analyzed the dataset using a simple random-effects model estimated by restricted maximum likelihood and with standard errors estimated with the Knapp-Hartung adjustment. However, note that a best-practice meta-analysis would account for the clustering via, for example, robust estimation (Hedges et al., 2010) or multilevel modeling, or a combination (Pustejovsky \& Tipton, 2021).
} 


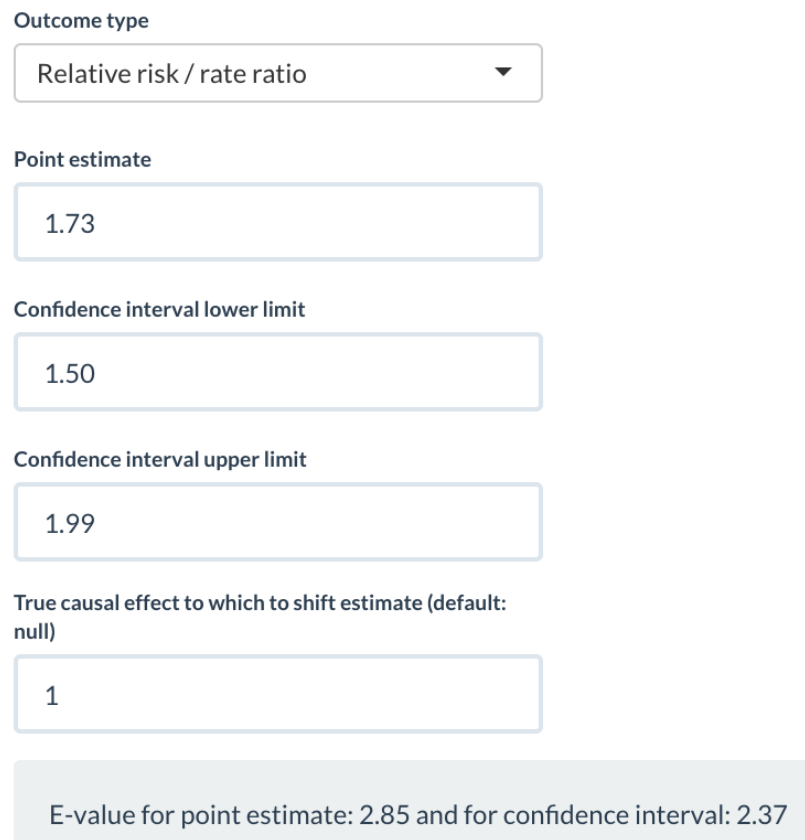

Alternatively, we could use the R package EValue by passing estimates from the meta-analysis object, meta. See also the standard $\mathrm{R}$ documentation and package vignettes for details (Mathur et al., 2018)).

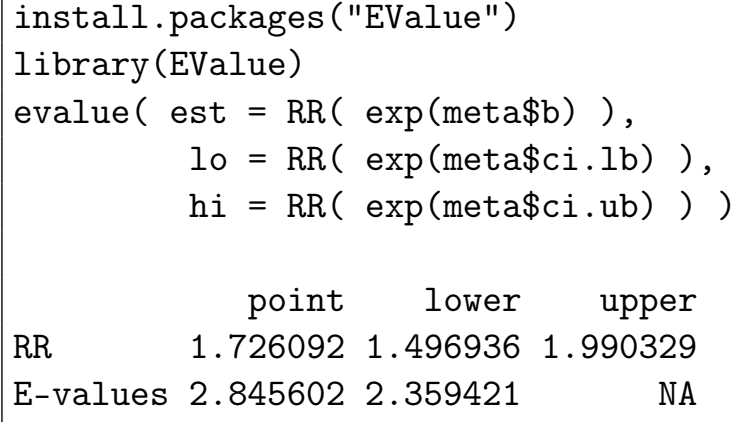

Throughout this example, the numerical values produced by the $\mathrm{R}$ package (and as reported in the main text) differ slightly from those produced by the website. This occurs because we used rounded values as inputs to the website but used exact values as inputs to the $\mathrm{R}$ function.

2.2. Estimating the percentage of effects above $R R=1.1$ and the strength of homogeneous confounding required to reduce the percentage to less than $15 \%$

The sensitivity analyses we conducted above describe evidence strength in the meta-analysis in terms of only its pooled point estimate. To additionally characterize heterogeneity across the studies' true causal effects, we switch to the website tab called "Sensitivity analysis for the proportion of meaningfully strong effects". This tab has two options: "Robust estimation (homogeneous bias across studies)" and "Parametric estimation (allows heterogeneous bias)". The analyses shown in the main text all consider homogeneous bias across studies, so we use the "Robust" tab.

First, we are prompted to upload our meta-analysis dataset as a .csv file. This dataset should contain 
at least two columns: one containing studies' point estimates on the log-relative risk scale, and one containing their variance estimates (i.e., squared standard errors). The dataset must have a single header row containing the variable names, like this:

\begin{tabular}{r|r|r|}
\multicolumn{2}{|l|}{ yi } & \multicolumn{1}{l|}{ vi } \\
\hline $\mathbf{1}$ & 0.385262400790645 & 0.000731026514084949 \\
\hline $\mathbf{2}$ & 0.425267735404344 & 0.00938935228099761 \\
\hline $\mathbf{3}$ & 0.22314355131421 & 0.00684180739201936 \\
\hline $\mathbf{4}$ & 0.198850858745165 & 0.144504220605036 \\
\hline $\mathbf{5}$ & 0.65232518603969 & 0.0233244877241552 \\
\hline $\mathbf{6}$ & 0.22314355131421 & 0.0346130484520095 \\
\hline $\mathbf{7}$ & 0.802001585472027 & 0.0399853247689023 \\
\hline
\end{tabular}

We upload the dataset to the website and also input the column names for the point estimates and the variance estimates (here, "yi" and "vi").

Next we fill out the section "Specify sensitivity parameters and thresholds". We select a scale (logrelative risk or relative risk) on which to input the bias factor in each study and the threshold we have chosen to represent a meaningfully strong effect size; for this example we use the relative risk scale. To estimate the percentage of studies with meaningfully strong population effects prior to correction for unmeasured confounding, we type "1" for the bias factor in each study (because multiplying a relative risk by a bias factor of 1 would leave it unchanged, representing no bias due to unmeasured confounding).

At the same time, we can also estimate the strength of homogeneous confounding that would be required to reduce this percentage of meaningfully strong effects to less than $15 \%$. To do so, we also type in our chosen threshold of $R R=1.1$, the proportion below which the proportion of meaningfully strong effects is to be reduced (0.15), and we select the tail of effects we want to consider ("above" because we want to consider effects above, rather than below, $R R=1.1$ ). The number of bootstrap iterates is used when estimating confidence intervals and defaults to 1,000 , which is what is used in this example.

\footnotetext{
${ }^{b}$ As indicated in the relevant input box in the website, the studies' point estimates and variances should be provided on the log-relative risk scale regardless of whether the sensitivity parameters are provided on the relative risk or log-relative scale. This is because the meta-analysis should be conducted using estimates on the log scale and because variances of relative risks are usually estimated on the log scale. For meta-analyses that were conducted with other types of effect sizes (e.g., odds ratios with a common outcome or standardized mean differences), the estimates and variances should first be converted to the log-relative risk scale, for example using the function convert_measures in the $\mathrm{R}$ package EValue.
} 


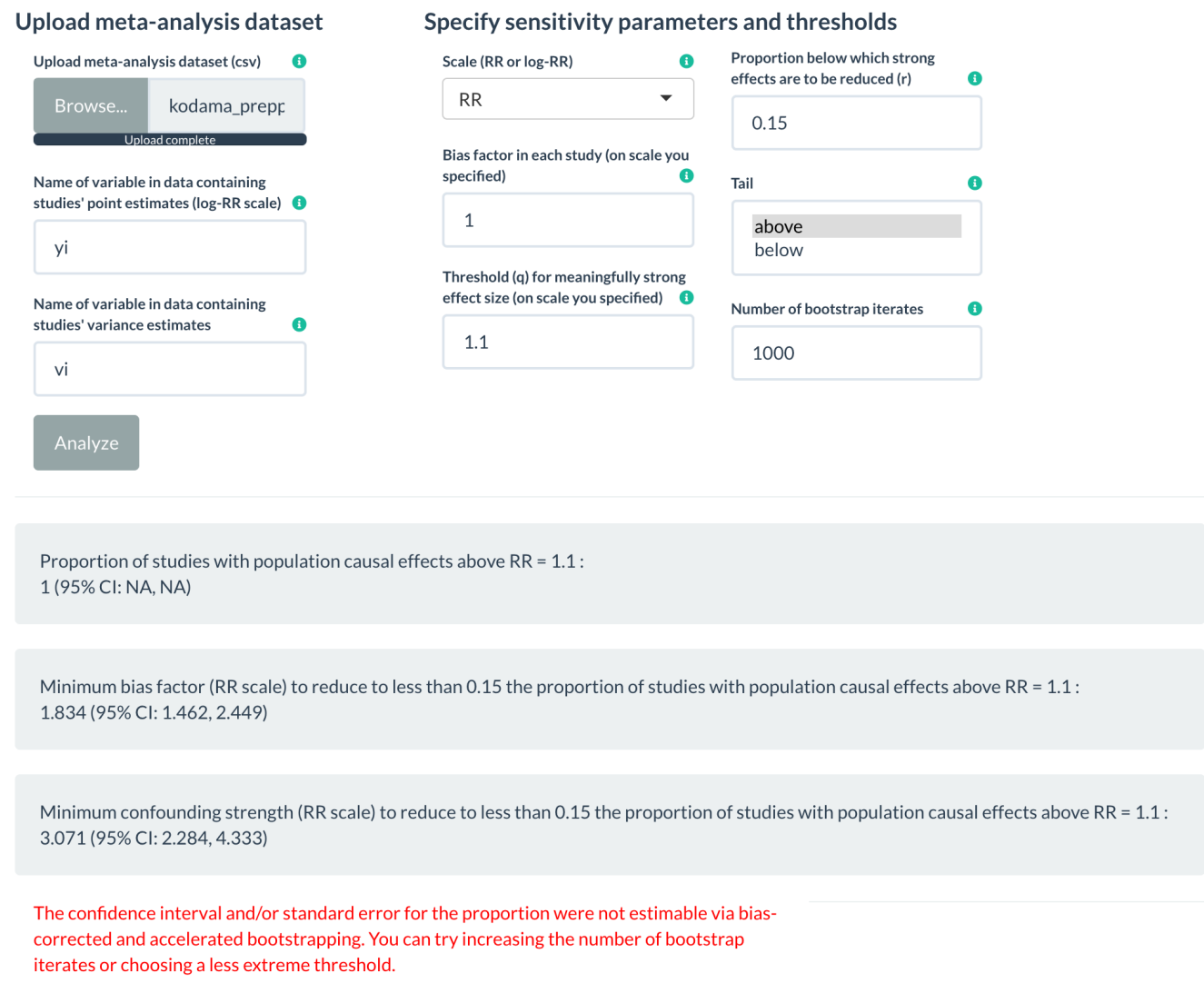

This analysis may take a minute or so to run, after which the metrics we want to estimate appear in the grey boxes. The output in the first box indicates that, prior to correction for unmeasured confounding, we estimate that the percentage of studies with meaningfully strong population effects $(R R>1.1)$ is nearly $100 \%$. There is no confidence interval ("NA") because, per the red message at the bottom of the screen, we have chosen an extremely high threshold compared to the distribution of effects in the meta-analysis, and this can make it impossible to estimate confidence intervals. The output in the second box is not discussed in this tutorial paper, but is described in the instructions of the website. The output in the third box indicates that to bring this percentage of meaningfully strong effects below 15\%, homogeneous unmeasured confounding associations with both higher aerobic capacity and lower all-cause mortality of $R R=3.07$ (95\% CI: $[2.28,4.33])$ each could suffice, but weaker homogeneous confounding could not.

Alternatively, we could use the $\mathrm{R}$ package as follows:

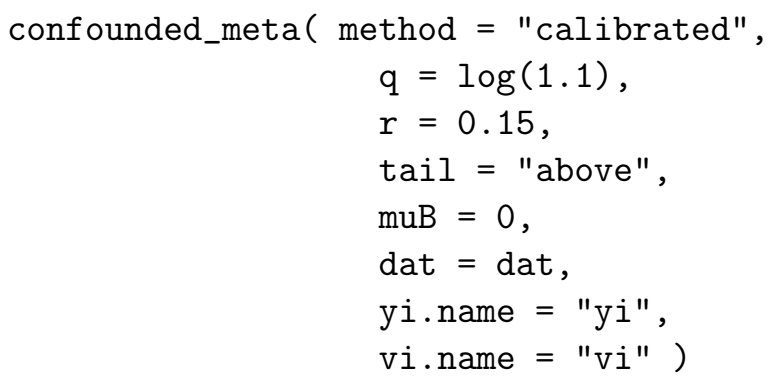

which yields the following output: 
The confidence interval and/or standard error for the proportion were not estimable via bias-corrected and accelerated bootstrapping. You can try increasing R.
Value
Est
SE CI.10
CI.hi
1 Prop 1.000000
NA NA NA
2 Tmin $1.833881 \quad 0.2452685 \quad 1.490533 \quad 2.420839$
3 Gmin $3.070504 \quad 0.5084525 \quad 2.3456094 .275461$

Because these confidence intervals are constructed by bootstrapping, they differ slightly from those provided on the website. In the main text, we report the confidence intervals given by the $\mathrm{R}$ package.

To consider heterogeneous bias as described in the main text, we can use the website tab "Parametric estimation (allows heterogeneous bias)". Here, rather than uploading the meta-analysis dataset, we input 4 estimates from our meta-analysis: the point estimate, its estimated variance (i.e., squared standard error, which can be found in the R output shown in Section 2.1], the estimated heterogeneity (called " $\mathrm{tau}$ '2" in the R output), and the estimated variance of the heterogeneity estimate. On the right side of the website, we again input the sensitivity parameters as we did in Section 2.2, but now, to characterize our assumed degree of heterogeneity in the confounding bias across studies, we specify what proportion of the observed heterogeneity (of $\widehat{\tau}_{c}^{2}=0.04$ ) is assumed to be due to variation in confounding bias rather than to genuine heterogeneity in studies' causal effects. If this proportion is 0 , then confounding is assumed to be of homogeneous severity across studies. The closer the proportion is to 1 , the more the severity of confounding is assumed to differ across studies.

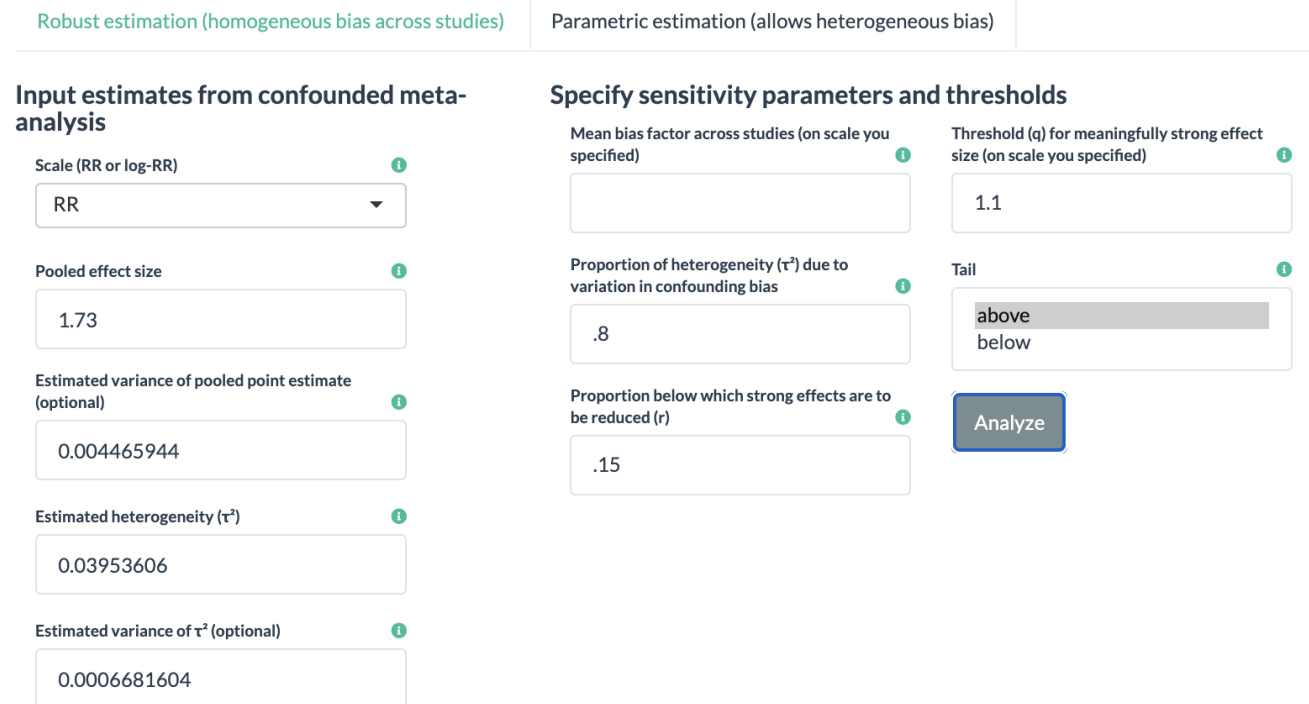

Proportion of studies with population causal effects above $R R=1.1$ NA $(95 \% \mathrm{Cl}: \mathrm{NA}, \mathrm{NA})$

Minimum bias factor (RR scale) to reduce to less than 0.15 the proportion of studies with population causal effects above $R R=1.1$ : 1.725 (95\% Cl: $1.168,2.282)$

Minimum confounding strength (RR scale) to reduce to less than 0.15 the proportion of studies with population causal effects above RR $=1.1$ : $2.842(95 \% \mathrm{Cl}: 1.675,4.01)$ 


\section{Supplemental figures FOR The APPlied EXAMPle}

\section{Study}

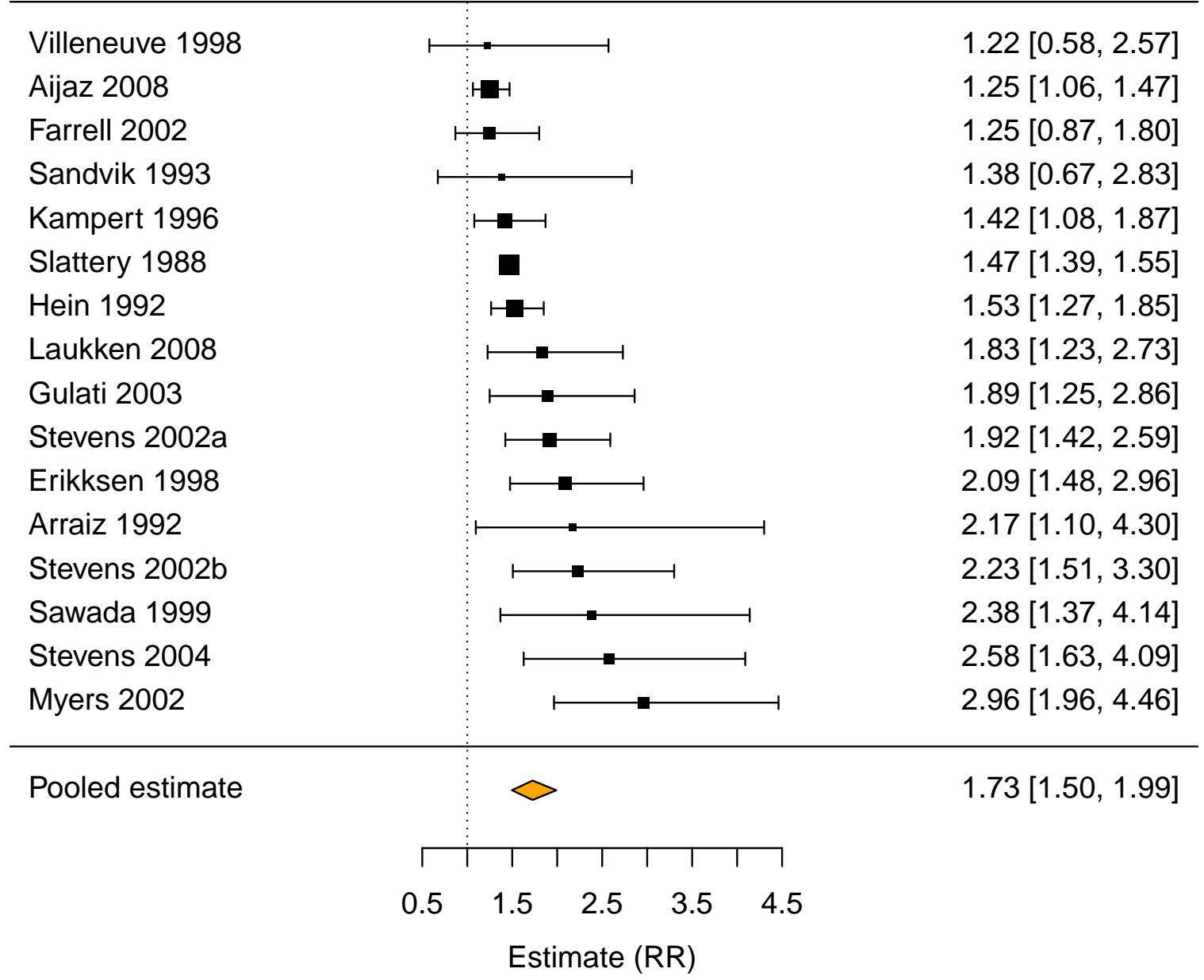

Supplemental Figure 1: Forest plot of point estimates (RR) and uncorrected pooled estimate in our reanalysis of Kodama et al. (2009)'s meta-analysis on lower aerobic capacity and mortality. Studies are ordered from largest to smallest point estimate. 


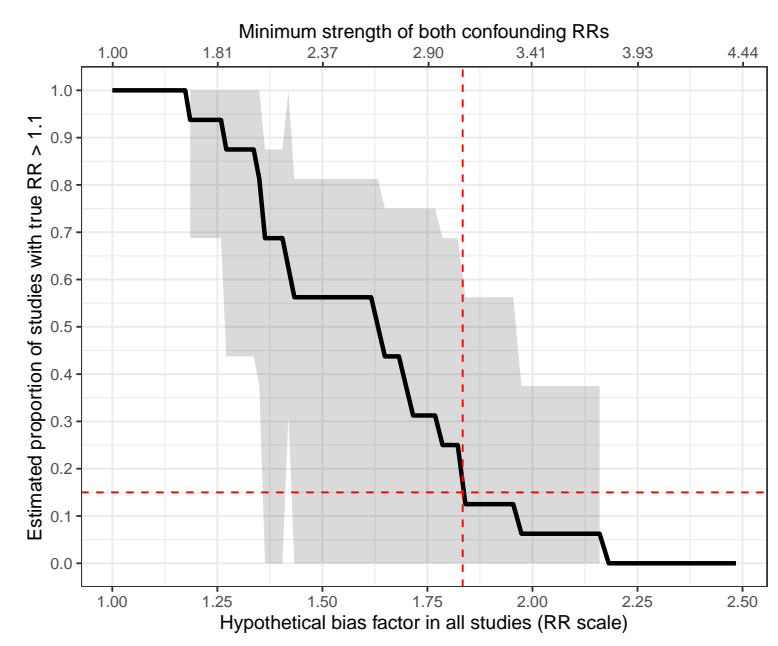

(a)

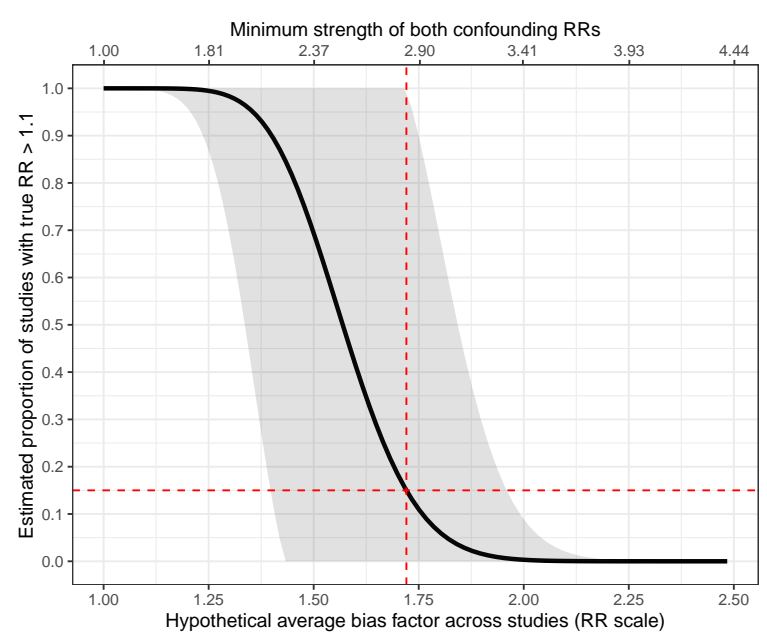

(b)

Supplemental Figure 2: (a) In sensitivity analyses considering homogeneous bias across studies with nonparametric estimation, the estimated proportion of studies with meaningfully strong causal effects $(R R>1.1)$ as a function of the multiplicative bias in all studies (lower $x$-axis) or, equivalently, the confounding strength in all studies (upper $x$-axis). Horizontal dashed line: the threshold $(r)$ at which only 15\% of effects are meaningfully strong. Vertical dashed line: the confounding strength required to reduce to less than 0.15 the proportion of meaningfully strong effects $(\widehat{G}(r=0.15, q=\log (1.1))$. (b) Counterpart for sensitivity analyses considering heterogeneous bias across studies. The confidence intervals in this panel are estimated parametrically and may not perform well for values of the proportion that are less than 0.15 or greater than 0.85 (Mathur \& VanderWeele, 2020b).

\section{EMPIRICAL BENCHMARKS ON AGREEMENT BETWEEN NONRANDOMIZED AND RANDOMIZED STUDIES IN META-ANALYSES}

Although directly estimating the extent of confounding bias in meta-analyzed studies would be very difficult, several studies have addressed this issues indirectly by estimating the extent of agreement or disagreement between NRS and RS on the same topic. Most relevant to our discussion are meta-meta-analyses in which investigators sample existing meta-analyses that contain both NRS and RS, calculate metrics of agreement between the study designs for each meta-analysis, and then summarize these agreement metrics across meta-analyses. If, within a meta-analysis, the estimates from NRS were on average biased upward or downward due to systematic confounding, this would tend to decrease agreement between the two study designs. Critically, though, any such disagreements could reflect not only unmeasured confounding, but also other biases that might preferentially affect one study design (e.g., publication bias) as well as differing distributions of effect modifiers (e.g., populations, interventions, or outcome measures) between the study designs. Therefore, estimates from these meta-meta-analyses should not be interpreted as direct estimates of confounding bias, but rather of the aggregation of confounding bias plus any other systematic differences between study designs.

Supplemental Table 2 summarizes the results of 4 such meta-meta-analyses (Bun et al., 2020; Golder et al., 2011; Shikata et al., 2006; Ioannidis et al., 2001) that used ratio outcomes that were comparable to $R R$ s. When possible, we re-analyzed data (Mathur \& VanderWeele, 2020a) to estimate the percentage of discrepancy ratios (i.e., the pooled $R R$ in NRS divided by that in RS) that were 
more extreme than various thresholds (e.g., 1.25). In the 2 meta-meta-analyses for which we could obtain study-level estimates to conduct these analyses, about $50 \%$ of meta-analyses had discrepancy ratios greater than 1.25 or less than 0.80 , but few $(\leq 10 \%)$ had discrepancy ratios greater than 2 or less than 0.50. Again, these estimates potentially reflect multiple systematic differences between the study designs, not only unmeasured confounding bias.

Outside the context of meta-analyses, other studies have compared NRS to RS while more stringently minimizing systematic differences in populations, interventions, and outcomes. In the ongoing RCTDUPLICATE project, existing medical RS are being "replicated" in NRS using claims data (Franklin et al. 2020). In the first 10 replications, one of 10 study pairs had a discrepancy with $p<0.05$ (Franklin et al. 2020), and we estimated that none (0\%) of the discrepancy ratios were greater than 1.25 or less than 0.80 . These discrepancies appear to be less than seen in the meta-meta-analyses, perhaps partly reflecting extensive confounding control in the NRS conducted by RCT-DUPLICATE. A few studies have even randomly assigned subjects to participate in either an observational study or an RCT, which would eliminate average discrepancies in the characteristics of subjects participating in each study design (Shadish et al., 2008, 2011). We encourage further empirical work comparing NRS to RS, including in meta-analyses and accounting for differing distributions of suspected effect modifiers (Dahabreh et al., 2020; Mathur \& VanderWeele, 2021).

\footnotetext{
${ }^{c}$ Our numerical results and qualitative interpretations sometimes disagree considerably with those reported in the meta-meta-analyses themselves for two reasons. First, some of the meta-meta-analyses reported much larger percentages of extreme discrepancy ratios than those we report. Those analyses had estimated these percentages by simply counting the number of estimated ratios that were above or below various thresholds. This method does not account for the substantial statistical error associated with ratio estimates, leading to substantially overestimated percentages of extreme ratios. We instead used methods that correct for this statistical error (Mathur \& VanderWeele 2020a), leading us to estimate smaller percentages of extreme ratios. Second, some meta-meta-analyses concluded that there was little disagreement based on the average discrepancy ratio across meta-analyses, but such results could occur even if discrepancies were extreme but occurred in different directions in different meta-analyses.
} 


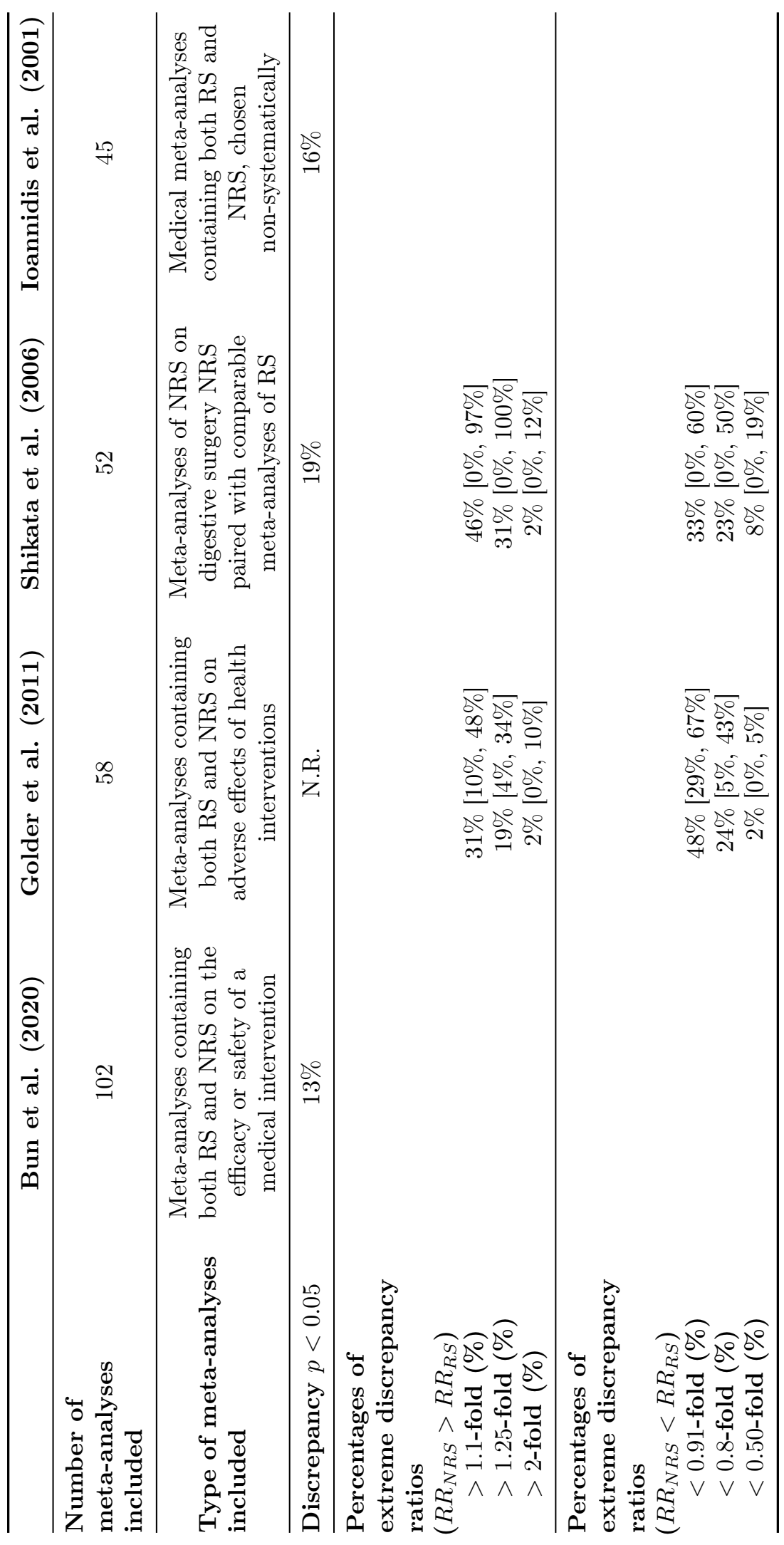

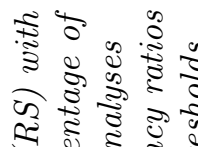

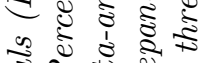

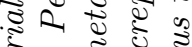

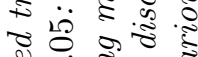
๘ 0 है ई సै 2 हो हूँ वे

व हु के

है के एँ

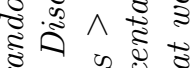

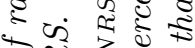
造

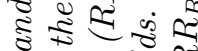

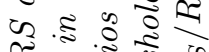
द. चू के है हाँ गँ

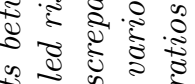
సٓ

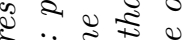
हू के है के की ज्ञ के डू हूँ क्ष 8 उ

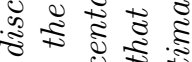
ช. है क्षे के

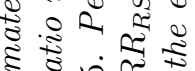
ह5 $102 \pi$ के के के को

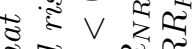

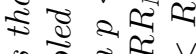
के

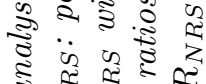
की I 20 व

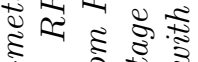
i. है उ. के क का ए

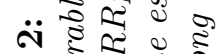

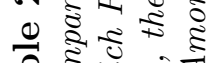
ส है ते को

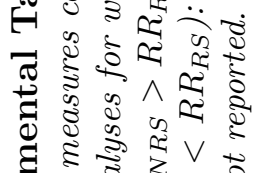

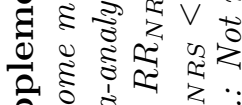
की 


\section{ADDITIONAL SENSITIVITY ANALYSIS FORMULAS}

\section{1. $\widehat{T}(r, q)$ and $\widehat{G}(r, q)$ with log-normal bias}

The expressions given the main text for $\widehat{T}(r, q)$ and $\widehat{G}(r, q)$ apply if the confounded pooled estimate is apparently causative $\left(\widehat{\mu}^{c}>0\right)$. If instead the confounded pooled estimate is apparently preventive $\left(\widehat{\mu}^{c}<0\right)$, the expression for $\widehat{G}(r, q)$ in terms of $\widehat{T}(r, q)$ remains the same, but $\widehat{T}(r, q)$ itself becomes:

$$
\begin{aligned}
\widehat{T}(r, q) & =\exp \left\{q-\widehat{\mu}^{c}-\Phi^{-1}(r) \sqrt{\widehat{\tau}_{c}^{2}-\sigma_{B^{*}}^{2}}\right\} \\
\widehat{\operatorname{SE}}(\widehat{T}(r, q)) & =\exp \left\{q-\widehat{\mu}^{c}-\Phi^{-1}(r) \sqrt{\widehat{\tau}_{c}^{2}-\sigma_{B^{*}}^{2}}\right\} \sqrt{\widehat{\operatorname{Var}}\left(\widehat{\mu}^{c}\right)+\frac{\widehat{\operatorname{Var}}\left(\widehat{\tau}_{c}^{2}\right)\left(\Phi^{-1}(r)\right)^{2}}{4\left(\widehat{\tau}_{c}^{2}-\sigma_{B^{*}}^{2}\right)}}
\end{aligned}
$$

These expressions, like those given in the main text, are straightforward generalizations of those given in Section 4.2 of Mathur \& VanderWeele (2020b) for homogeneous bias.

\subsection{Sensitivity analysis with weakened assumptions on the bias distribution}

Here we provide a somewhat more technical explanation of how the E-value for the pooled estimate (VanderWeele \& Ding, 2017; Mathur \& VanderWeele, 2020b) and the nonparametric estimates $\widehat{P}_{>q}, \widehat{T}(r, q)$, and $\widehat{G}(r, q)$ (Mathur \& VanderWeele, 2020a) can be calculated and interpreted under weakened assumptions about the distribution of bias. For the $i^{\text {th }}$ meta-analyzed study, let $\widehat{\theta}_{i}^{c}$ denote its confounded estimate, $\theta_{i}^{t}$ its population causal effect, $B_{i}^{*}$ its bias, and $\epsilon_{i}$ its statistical error, such that $\widehat{\theta}_{i}^{c}=\theta_{i}^{t}+B_{i}^{*}+\epsilon_{i}$. As in the main text, we use the superscript ${ }^{c}$ to denote confounded estimates and parameters and the superscript ${ }^{t}$ to denote their true (i.e., causal) counterparts, with $\widehat{\mu}^{c}$ denoting the confounded pooled $\log -R R$. Let $k$ denote the number of studies in the meta-analysis.

\subsubsection{E-value for the pooled estimate and confidence interval}

First, as discussed in the main text, the E-value calculated using the confounded pooled estimate is $\exp \left(\widehat{\mu}^{c}\right)+\sqrt{\exp \left(\widehat{\mu}^{c}\right)\left(\exp \left(\widehat{\mu}^{c}\right)-1\right)}$. All the considerations discussed below also apply when calculating the E-value for the confidence interval limit. The E-value for the pooled estimate and confidence interval can be interpreted without assumptions on the distribution of the population causal effects or the distribution of bias across the population causal effects provided that:

- Condition (i). Any distributional assumptions of the meta-analysis model are fulfilled. This implies that if the population confounded effects, $\theta_{i}^{c}$, are not normal, the meta-analysis must be conducted using an approach that does not make this distributional assumption (Hedges et al. 2010: Pustejovsky \& Tipton, 2021).

- Condition (ii). The bias in each study is independent of its population causal effect, $\theta_{i}^{t}$, and its standard error. That is, studies with larger population causal effects cannot have systematically 
more or less bias than studies with smaller population causal effects, and more precise studies cannot have systematically more or less bias than less precise studies.

Along with standard assumptions of meta-analysis, these conditions imply that $\widehat{\mu}^{c}$ is consistent in $k$ for $\mu^{c}$. This consistency implies that the E-value calculated using $\widehat{\mu}^{c}$ is itself consistent for the population E-value that would be calculated using the confounded population mean $\mu^{c}$. Specifically, standard meta-analysis methods based on inverse-variance weighting require studies' population effects to be independent of their standard errors. If we thus make the standard assumption that $\theta_{i}^{t} \amalg \epsilon_{i}$ and additionally assume that Condition (ii) holds (i.e., $B_{i}^{*} \amalg \epsilon_{i}$ and $B_{i}^{*} \amalg \theta_{i}^{t}$ ), then these assumptions imply that $\theta_{i}^{c}=\theta_{i}^{t}+B_{i}^{*} \amalg \epsilon_{i}$. Then, under standard regularity conditions, $\widehat{\mu}^{c}$ is consistent for $\mu^{c}$ without further assumptions on the distribution of $\theta_{i}^{c}=\theta_{i}^{t}+B_{i}^{*}$, subject to the choice of a meta-analysis model that fulfills Condition (i).

Note that even if $\theta_{i}^{t}$ are assumed to be normal, the first condition is still necessary to accommodate the possibility of non-normal distributions of bias. In practice, diagnostic plots and tests could be used to check the normality assumption (Hardy \& Thompson, 1998, Wang \& Lee, 2020). If normality appears to hold, then a standard parametric meta-analysis could still be used. Using parametric estimation facilitates estimating the standard error of $\widehat{\tau}_{c}^{2}$, which is used when constructing parametric confidence intervals for $\widehat{P}_{>q}$.

\subsection{2 $\widehat{P}_{>q}, \widehat{G}(r, q)$, and $\widehat{T}(r, q)$}

To estimate $\widehat{P}_{>q}$ under homogeneous bias $B^{*}$, the method of Mathur \& VanderWeele (2020a) involves calculating a bias-corrected "calibrated" estimate (Wang \& Lee, 2019) for each meta-analyzed study, $\widetilde{\theta}_{i}$, and then calculating the sample proportion of $\widetilde{\theta}_{i}$ that are above $q$ as follows. Let $\tau_{t}^{2}=\operatorname{Var}\left(\theta^{t}\right)$ denote the heterogeneity of the population causal effects and $\widehat{\tau}_{t}^{2}$ its sample estimate based on the confounded meta-analysis, whose form is given below. Define a generic calibrated estimate as the function:

$$
\widetilde{\theta}_{i}(b, v)=\widehat{\mu}^{c}-b+\sqrt{\frac{v}{\widehat{\tau}_{c}^{2}+\widehat{\sigma}_{i}^{2}}}\left(\widehat{\theta}_{i}^{c}-\widehat{\mu}^{c}\right)
$$

Assuming homogeneous bias of magnitude $B^{*}$, the calibrated estimates $\widetilde{\theta}_{i}\left(B^{*}, \widehat{\tau}_{c}^{2}\right)$ approximately match the first two moments of the marginal distribution of population effects (Wang \& Lee, 2019). The proportion of meaningfully strong effects can then be estimated as (Mathur \& VanderWeele, 2020a): d

$$
\widehat{P}_{>q}(b, v)=\frac{1}{k} \sum_{i=1}^{k} \mathbb{1}\left\{\widetilde{\theta}_{i}\left(B^{*}, \widehat{\tau}_{c}^{2}\right)>q\right\}
$$

The following lemma and corollary show that, under Conditions (i) and (ii) given in Section 5.2.1, the calibrated estimates could instead be calculated using an estimated lower or upper bound on $\tau_{t}^{2}$. The lower bound on $\tau_{t}^{2}$ is attained when the variance of the bias $\left(\sigma_{B^{*}}^{2}\right)$ is the most that the meta-analyst

\footnotetext{
${ }^{d}$ Here, in contrast to Mathur \& VanderWeele $\left(2020\right.$ a) we adopt the more explicit notation " $\widehat{P}_{>q}(b, v)$ " to emphasize the dependence of this estimate on the choice of $b$ and $v$ used to calculate the calibrated estimates.
} 
believes is plausible (called $U B\left(\sigma_{B^{*}}^{2}\right)$ ); the upper bound on $\tau_{t}^{2}$ is attained when the bias is homogeneous. The following results show that the calibrated estimates calculated using the lower bound on $\tau_{t}^{2}$ and assuming a mean bias of $\mu_{B^{*}}$ are $\widetilde{\theta}_{i}\left(\mu_{B^{*}}, \widehat{\tau}_{c}^{2}-U B\left(\sigma_{B^{*}}^{2}\right)\right)$ and are underdispersed compared to the distribution of the causal population effects. Conversely, the calibrated estimates calculated using the upper bound on $\tau_{t}^{2}$ are $\widetilde{\theta}_{i}\left(\mu_{B^{*}}, \widehat{\tau}_{c}^{2}\right)$ and are overdispersed compared to the distribution of the causal population effects. This leads to estimates $\widehat{P}_{>q}$ that are typically, though not always, overestimates or underestimates depending on whether the bias-corrected pooled estimate is above or below $q$ (Supplemental Table 3). These results could be used to calculate estimates, $\widehat{P}_{>q}(b, v)$, that are considered conservative in the context of whether one is claiming that a meta-analysis is robust or sensitive to unmeasured confounding.

Because $\widehat{T}(r, q)$ and $\widehat{G}(r, q)$ are simply roots in $b$ of the function $\widehat{P}_{>q}(b, v)$, they can also be interpreted similarly. For example, suppose $\widehat{\mu}^{c}=\log (2)$ and we estimate the severity of homogeneous bias (i.e., $\sigma_{B^{*}}^{2}=0$, so $\left.\widehat{\tau}_{t}^{2}=\widehat{\tau}_{c}^{2}\right)$ required to reduce the proportion of causal effects above $q=\log (1.1)$ to $r=0.15$. We might thus obtain $\widehat{T}(r, q)=1.5$, which is equivalent to estimating that $\widehat{P}_{>q}\left(\log (1.5), \widehat{\tau}_{c}^{2}\right)=0.15$. With this amount of bias, the bias-corrected mean is $\widehat{\mu}^{t}=\widehat{\mu}^{c}-\log (\widehat{T}(r, q)) \approx \log (1.33)$. Thus, $\widehat{\mu}^{t}>q$, so by consulting the third row and first column of Supplemental Table 3, we can conclude that the estimate $\widehat{P}_{>q}\left(\log (1.5), \widehat{\tau}_{c}^{2}\right)=0.15$ would typically underestimate the true proportion of meaningfully strong effects if in fact the bias were heterogeneous across studies. Equivalently, our estimate $\widehat{T}(r, q)=1.5$ is conservative in the sense that if the bias were in fact heterogeneous, then we would typically expect that at least $15 \%$ of effects would remain meaningfully strong.

\begin{tabular}{lll}
\hline & $\widehat{\mu}^{t}>q$ & $\widehat{\mu}^{t}<q$ \\
\hline$\widehat{P}_{>q}\left(\mu_{B^{*}}, \widehat{\tau}_{c}^{2}-U B\left(\sigma_{B^{*}}^{2}\right)\right)$ & Overestimate & Underestimate \\
$\widehat{P}_{<q}\left(\mu_{B^{*}}, \widehat{\tau}_{c}^{2}-U B\left(\sigma_{B^{*}}^{2}\right)\right)$ & Underestimate & Overestimate \\
$\widehat{P}_{>q}\left(\mu_{B^{*}}, \widehat{\tau}_{c}^{2}\right)$ & Underestimate & Overestimate \\
$\widehat{P}_{<q}\left(\mu_{B^{*}}, \widehat{\tau}_{c}^{2}\right)$ & Overestimate & Underestimate \\
\hline
\end{tabular}

Supplemental Table 3: Two methods of calculating $\widehat{P}_{>q}$ and $\widehat{P}_{<q}$ (i.e., the proportion of population causal effects below $q$ ) and the conditions under which they are typically overestimates or underestimates compared to the estimate that would be obtained using the true heterogeneity $\tau_{t}^{2}$. These results apply regardless of the sign of $\widehat{\mu}^{c}$ (i.e., whether it is apparently causative or apparently preventive); as in Mathur 6 VanderWeele (2020b), we consider bias that operates on average away from the null with the convention $\mu_{B^{*}}>0$ regardless of the sign of $\widehat{\mu}^{c}$, such that $\widehat{\mu}^{t}$ estimates $\mu^{t}$ and is equal to $\widehat{\mu}^{c}-\mu_{B^{*}}$ for $\widehat{\mu}^{c}>0$ and $\widehat{\mu}^{c}+\mu_{B^{*}}$ for $\widehat{\mu}^{c}<0$.

The following lemma and corollary establish the claims made in Supplemental Table 3 .

Lemma 1 (Underdispersed calibrated estimates). Suppose $\mu_{B^{*}}=E\left[B^{*}\right]$ is considered known and that $\sigma_{B^{*}}^{2}$ is unknown, but has a known upper bound $U B\left(\sigma_{B^{*}}^{2}\right)$. Assume that $\operatorname{Corr}\left(\theta_{i}^{t}, B_{i}^{*}\right)=0$. If the calibrated estimates are calculated as:

$$
\widetilde{\theta}_{i}\left(\mu_{B^{*}}, \widehat{\tau}_{c}^{2}-U B\left(\sigma_{B^{*}}^{2}\right)\right)=\widehat{\mu}^{c}-\mu_{B^{*}}+\sqrt{\frac{\widehat{\tau}_{c}^{2}-U B\left(\sigma_{B^{*}}^{2}\right)}{\widehat{\tau}_{c}^{2}+\widehat{\sigma}_{i}^{2}}}\left(\widehat{\theta}_{i}^{c}-\widehat{\mu}^{c}\right)
$$

then $E\left[\widetilde{\theta}_{i}\left(\mu_{B^{*}}, \widehat{\tau}_{c}^{2}-U B\left(\sigma_{B^{*}}^{2}\right)\right)\right]$ approximately matches the expectation of the distribution of population 
causal effects, and the calibrated estimates are underdispersed in the sense that $\operatorname{Var}\left(\widetilde{\theta}_{i}\left(\mu_{B^{*}}, \widehat{\tau}_{c}^{2}-U B\left(\sigma_{B^{*}}^{2}\right)\right)\right)$ is consistent for a value that is at most $\tau_{t}^{2}$.

Proof. Regarding the first moment, we have $E\left[\widetilde{\theta}_{i}\left(\mu_{B^{*}}, \widehat{\tau}_{c}^{2}-U B\left(\sigma_{B^{*}}^{2}\right)\right)\right]=\mu^{c}-\mu_{B^{*}}$. Regarding the second moment, first note that $\tau_{t}^{2}=\tau_{c}^{2}-\sigma_{B^{*}}^{2}$, so $\tau_{t}^{2}$ is bounded by $\tau_{c}^{2}-U B\left(\sigma_{B^{*}}^{2}\right) \leq \tau_{t}^{2} \leq \tau_{c}^{2}$. (The lower bound, $\tau_{c}^{2}-\sigma_{B^{*}}^{2}$, is attained when the bias explains the maximum plausible amount of the observed heterogeneity; the upper bound, $\tau_{c}^{2}$, is attained when the bias explains none of the observed heterogeneity.) $\left.\right|^{e}$ Let " $\underset{k \rightarrow \infty}{\stackrel{p}{\longrightarrow}}$ " denote convergence in probability in $k$. By invoking Conditions (i) and (ii) so that $\widehat{\mu}^{c}$ and $\widehat{\tau}_{c}^{2}$ are each consistent, we have:

$$
\begin{aligned}
\operatorname{Var}\left(\widetilde{\theta}_{i}\left(\mu_{B^{*}}, \widehat{\tau}_{c}^{2}-U B\left(\sigma_{B^{*}}^{2}\right)\right)\right) & \underset{k \rightarrow \infty}{\stackrel{p}{\longrightarrow}} \frac{\tau_{c}^{2}-U B\left(\sigma_{B^{*}}^{2}\right)}{\tau_{c}^{2}+\sigma_{i}^{2}} \times \operatorname{Var}\left(\widehat{\theta}_{i}^{c}\right) \\
& =\tau_{c}^{2}-U B\left(\sigma_{B^{*}}^{2}\right) \\
& \leq \tau_{t}^{2}
\end{aligned}
$$

Corollary 1 (Overdispersed calibrated estimates). Under the same assumptions given above, if the calibrated estimates are calculated as:

$$
\tilde{\theta}_{i}\left(\mu_{B^{*}}, \widehat{\tau}_{c}^{2}\right)=\widehat{\mu}^{c}-\mu_{B^{*}}+\sqrt{\frac{\widehat{\tau}_{c}^{2}}{\widehat{\tau}_{c}^{2}+\widehat{\sigma}_{i}^{2}}}\left(\widehat{\theta}_{i}^{c}-\widehat{\mu}^{c}\right)
$$

then $E\left[\widetilde{\theta}_{i}\left(\mu_{B^{*}}, \widehat{\tau}_{c}^{2}\right)\right]$ approximately matches the expectation of the distribution of population causal effects and the calibrated estimates are overdispersed in the sense that $\operatorname{Var}\left(\widetilde{\theta}_{i}\left(\mu_{B^{*}}, \widehat{\tau}_{c}^{2}\right)\right)$ is consistent for a value that is at least $\tau_{t}^{2}$.

Proof. This follows immediately from the bound $\tau_{t}^{2} \leq \tau_{c}^{2}$, attained when $\sigma_{B^{*}}^{2}=0$.

The overdispersed calibrated estimates in Eq. (S.5) are equivalent to the calibrated estimates calculated by assuming homogeneous bias, $\widetilde{\theta}_{i}\left(B^{*}, \widehat{\tau}_{c}^{2}\right)$, when the homogeneous bias $B^{*}$ is set equal to $\mu_{B^{*}}$.

\section{REFERENCES}

Bun, R.-S., Scheer, J., Guillo, S., Tubach, F., \& Dechartres, A. (2020). Meta-analyses frequently pooled different study types together: a meta-epidemiological study. Journal of Clinical Epidemiology, $118,18-28$.

${ }^{e}$ In principle, the assumption that the population causal effects are uncorrelated with their biases could be relaxed by specifying that correlation or an upper bound on the correlation. Then we have $\tau_{t}^{2} \geq \tau_{c}^{2}-U B\left(\sigma_{B^{*}}^{2}\right)-$ $2 \operatorname{Corr}\left(\theta_{i}^{t}, B_{i}^{*}\right) \sqrt{\widehat{\tau}_{c}^{2} \times U B\left(\sigma_{B^{*}}^{2}\right)}$, where the correlation could be replaced by its own upper bound. The calibrated estimates would then be $\widetilde{\theta}_{i}\left(\mu_{B^{*}}, \widehat{\tau}_{c}^{2}-U B\left(\sigma_{B^{*}}^{2}\right)-2 \operatorname{Corr}\left(\theta_{i}^{t}, B_{i}^{*}\right) \sqrt{\widehat{\tau}_{c}^{2} \times U B\left(\sigma_{B^{*}}^{2}\right.}\right)$. 
Dahabreh, I. J., Petito, L. C., Robertson, S. E., Hernán, M. A., \& Steingrimsson, J. A. (2020). Toward causally interpretable meta-analysis: Transporting inferences from multiple randomized trials to a new target population. Epidemiology, 31(3), 334-344.

Franklin, J. M., Patorno, E., Desai, R. J., Glynn, R. J., Martin, D., Quinto, K., .. others (2020). Emulating randomized clinical trials with nonrandomized real-world evidence studies: First results from the RCT DUPLICATE initiative. Circulation.

Golder, S., Loke, Y. K., \& Bland, M. (2011). Meta-analyses of adverse effects data derived from randomised controlled trials as compared to observational studies: Methodological overview. PLoS Medicine, 8(5), e1001026.

Hardy, R. J., \& Thompson, S. G. (1998). Detecting and describing heterogeneity in meta-analysis. Statistics in Medicine, 17(8), 841-856.

Hedges, L. V., Tipton, E., \& Johnson, M. C. (2010). Robust variance estimation in meta-regression with dependent effect size estimates. Research Synthesis Methods, 1(1), 39-65.

Ioannidis, J. P., Haidich, A.-B., Pappa, M., Pantazis, N., Kokori, S. I., Tektonidou, M. G., ... Lau, J. (2001). Comparison of evidence of treatment effects in randomized and nonrandomized studies. Journal of the American Medical Association, 286(7), 821-830.

Kodama, S., Saito, K., Tanaka, S., Maki, M., Yachi, Y., Asumi, M., ... others (2009). Cardiorespiratory fitness as a quantitative predictor of all-cause mortality and cardiovascular events in healthy men and women: A meta-analysis. Journal of the American Medical Association, 301 (19), 2024-2035.

Mathur, M. B., Ding, P., Riddell, C. A., \& VanderWeele, T. J. (2018). Website and R package for computing E-values. Epidemiology, 29(5), e45.

Mathur, M. B., \& VanderWeele, T. J. (2020a). Robust metrics and sensitivity analyses for metaanalyses of heterogeneous effects. Epidemiology, 31(3), 356-358.

Mathur, M. B., \& VanderWeele, T. J. (2020b). Sensitivity analysis for unmeasured confounding in meta-analyses. Journal of the American Statistical Association, 115(529), 163-172.

Mathur, M. B., \& VanderWeele, T. J. (2021). Meta-regression methods to characterize evidence strength using meaningful-effect percentages conditional on study characteristics. Research Synthesis Methods. (Preprint retrieved from https://osf.io/bmtdq/)

Pustejovsky, J. E., \& Tipton, E. (2021). Meta-analysis with robust variance estimation: Expanding the range of working models. Prevention Science, 1-14.

Shadish, W. R., Clark, M. H., \& Steiner, P. M. (2008). Can nonrandomized experiments yield accurate answers? A randomized experiment comparing random and nonrandom assignments. Journal of the American Statistical Association, 103(484), 1334-1344.

Shadish, W. R., Galindo, R., Wong, V. C., Steiner, P. M., \& Cook, T. D. (2011). A randomized experiment comparing random and cutoff-based assignment. Psychological Methods, 16(2), 179.

Shikata, S., Nakayama, T., Noguchi, Y., Taji, Y., \& Yamagishi, H. (2006). Comparison of effects in randomized controlled trials with observational studies in digestive surgery. Annals of Surgery, $244(5), 668$. 
VanderWeele, T. J., \& Ding, P. (2017). Sensitivity analysis in observational research: introducing the E-value. Annals of Internal Medicine, 167(4), 268-274.

Viechtbauer, W., et al. (2010). Conducting meta-analyses in R with the metafor package. Journal of Statistical Software, 36(3), 1-48.

Wang, C.-C., \& Lee, W.-C. (2019). A simple method to estimate prediction intervals and predictive distributions: Summarizing meta-analyses beyond means and confidence intervals. Research Synthesis Methods, 10(2), 255-266.

Wang, C.-C., \& Lee, W.-C. (2020). Evaluation of the normality assumption in meta-analyses. American Journal of Epidemiology, 189(3), 235-242. 